\title{
Search for supersymmetry in hadronic final states using $M_{\mathrm{T} 2}$ in pp collisions at $\sqrt{s}=7 \mathrm{TeV}$
}

\section{The CMS collaboration}

ABSTRACT: A search for supersymmetry or other new physics resulting in similar final states is presented using a data sample of $4.73 \mathrm{fb}^{-1}$ of pp collisions collected at $\sqrt{s}=7 \mathrm{TeV}$ with the CMS detector at the LHC. Fully hadronic final states are selected based on the variable $M_{\mathrm{T} 2}$, an extension of the transverse mass in events with two invisible particles. Two complementary studies are performed. The first targets the region of parameter space with medium to high squark and gluino masses, in which the signal can be separated from the standard model backgrounds by a tight requirement on $M_{\mathrm{T} 2}$. The second is optimized to be sensitive to events with a light gluino and heavy squarks. In this case, the $M_{\mathrm{T} 2}$ requirement is relaxed, but a higher jet multiplicity and at least one b-tagged jet are required. No significant excess of events over the standard model expectations is observed. Exclusion limits are derived for the parameter space of the constrained minimal supersymmetric extension of the standard model, as well as on a variety of simplified model spectra.

KEYwORDS: Hadron-Hadron Scattering 


\section{Contents}

1 Introduction 1

2 Definition of $M_{\mathrm{T} 2} \quad 2$

3 CMS detector 4

4 Samples and event selection $\quad 4$

$\begin{array}{llr}5 & \text { Search strategy } & 6\end{array}$

$\begin{array}{lll}6 & M_{\mathrm{T} 2} \text { analysis } & 7\end{array}$

6.1 Background prediction $\quad 8$

6.1.1 QCD multijet background $\quad 8$

6.1.2 $\mathrm{W}(\ell \nu)+$ jets and top-quark background $\quad 9$

$\begin{array}{ll}\text { 6.1.3 } \mathrm{Z}(\nu \bar{\nu})+\text { jets background } & 10\end{array}$

6.2 Results 11

$\begin{array}{lll}7 & M_{\mathrm{T} 2} b \text { analysis } & 11\end{array}$

$\begin{array}{lll}7.1 & \text { Background prediction and results } & 12\end{array}$

8 Statistical interpretation of the results and exclusion limits 13

$\begin{array}{ll}\text { 8.1 Exclusion limits in the CMSSM plane } & 15\end{array}$

$\begin{array}{ll}\text { 8.2 Exclusion limits for simplified model spectra } & 17\end{array}$

$\begin{array}{llr}9 & \text { Summary } & 18\end{array}$

$\begin{array}{lr}\text { The CMS collaboration } & 23\end{array}$

\section{Introduction}

A broad class of extensions of the standard model (SM) predict the existence of heavy colored particles that decay to hadronic final states accompanied by large missing transverse energy $\left(E_{\mathrm{T}}^{\text {miss }}\right)$. The best known of these scenarios is supersymmetry [1] (SUSY) with Rparity conservation. In this paper we present a search for such new physics in pp collisions collected with the Compact Muon Solenoid (CMS) detector at the Large Hadron Collider (LHC) at a center-of-mass energy of $7 \mathrm{TeV}$. The results are based on the data sample collected in 2011, corresponding to about $4.73 \mathrm{fb}^{-1}$ of integrated luminosity.

The search makes use of the "stransverse mass" variable $M_{\mathrm{T} 2}[2,3]$ to select new physics candidate events. $M_{\mathrm{T} 2}$ is the natural extension of the transverse mass $M_{\mathrm{T}}$ to the case where two colored supersymmetric particles ("sparticles") are pair-produced and 
both decay through a cascade of jets and possibly leptons to the lightest supersymmetric particle (LSP). The LSP is not visible in the detector and leads to a missing transverse momentum signature. Although $M_{\mathrm{T} 2}$ was originally introduced to derive the masses of sparticles involved in the cascade decay, we use it here as a discovery variable since it is sensitive to the presence of SUSY-like new physics. The distribution of $M_{\mathrm{T} 2}$ reflects the produced particle masses, which are much lighter for the SM background processes than for the SUSY processes. Hence, new physics is expected to appear as an excess in the tail of $M_{\mathrm{T} 2}$.

The analysis is based on two complementary approaches. A first approach, the " $M_{\mathrm{T} 2}$ analysis", targets events resulting from heavy sparticle production, characterized by large $E_{\mathrm{T}}^{\text {miss }}$, at least three jets, and large $M_{\mathrm{T} 2}$. The SM backgrounds in the signal region consist of $\mathrm{W}(\ell \nu)+$ jets, $\mathrm{Z}(\nu \bar{\nu})+$ jets, $\mathrm{t} \overline{\mathrm{t}}$, and single-top events (the last two will be referred to collectively as top-quark background), which are estimated from data-control regions and simulation. This analysis loses sensitivity if the squarks are heavy and the gluinos light, in which case the production is dominated by gluino-gluino processes. The gluinos give rise to three-body decays with relatively small $E_{\mathrm{T}}^{\text {miss }}$. Since the gluino decay is mediated by virtual squark exchange and the stop and sbottom are expected to be lighter than the first- and second-generation squarks, these events can be rich in b quarks. To increase the sensitivity to such processes, a second approach, the " $M_{\mathrm{T} 2} b$ analysis", is developed, in which the threshold on $M_{\mathrm{T} 2}$ defining the signal region is lowered. To suppress the QCD multijet background, we demand at least one b-tagged jet and place a stricter requirement on the jet multiplicity. The $M_{\mathrm{T} 2} b$ analysis provides a larger signal-to-background ratio in the region of heavy squarks and light gluinos and hence improves our sensitivity to this scenario.

This paper extends previous results of searches in fully hadronic final states from the CMS [4-7] and ATLAS [8-11] Collaborations. It is organized as follows: after a brief introduction to $M_{\mathrm{T} 2}$ and its salient properties in section 2, and a description of the CMS detector in section 3 , we present in section 4 the data samples used and the event selection. In section 5, the search strategy is presented. This strategy is applied to the $M_{\mathrm{T} 2}$ analysis in section 6 and to the $M_{\mathrm{T} 2} b$ analysis in section 7 . In these sections the background estimation methods are also discussed. We interpret the results in section 8 in the context of the constrained minimal supersymmetric standard model (CMSSM) as well as for a variety of simplified models. Finally, section 9 contains a summary.

\section{Definition of $M_{\mathrm{T} 2}$}

The variable $M_{\mathrm{T} 2}$ was introduced [2] to measure the mass of primary pair-produced particles in a situation where both ultimately decay into undetected particles (e.g., LSPs) leaving the event kinematics underconstrained. It assumes that the two produced sparticles give rise to identical types of decay chains with two visible systems defined by their transverse momenta $\vec{p}_{\mathrm{T}}^{\mathrm{vis}(i)}$, transverse energies $E_{\mathrm{T}}^{\mathrm{vis}(i)}$, and masses $m^{\mathrm{vis}(i)}$. They are accompanied by the unknown LSP transverse momenta $\vec{p}_{\mathrm{T}} \widetilde{\chi(i)}$. In analogy with the transverse mass used for 
the $\mathrm{W}$ boson mass determination [12], we can define two transverse masses $(i=1,2)$ :

$$
\left(M_{\mathrm{T}}^{(i)}\right)^{2}=\left(m^{\mathrm{vis}(i)}\right)^{2}+m_{\widetilde{\chi}}^{2}+2\left(E_{\mathrm{T}}^{\mathrm{vis}(i)} E_{\mathrm{T}}^{\widetilde{\chi}(i)}-\vec{p}_{\mathrm{T}}^{\mathrm{vis}(i)} \cdot \vec{p}_{\mathrm{T}}^{\widetilde{\chi}(i)}\right) .
$$

These have the property (as in W-boson decays) that, for the true LSP mass $m_{\tilde{\chi}}$, their distribution cannot exceed the mass of the parent particle of the decay and they present an endpoint at the value of the parent mass. The momenta $\vec{p}_{\mathrm{T}}^{\widetilde{\chi}(i)}$ of the invisible particles are not experimentally accessible individually. Only their sum, the missing transverse momentum $\vec{p}_{\mathrm{T}}^{\text {miss }}$, is known. Therefore, in the context of SUSY, a generalization of the transverse mass is needed and the proposed variable is $M_{\mathrm{T} 2}$. It is defined as

$$
M_{\mathrm{T} 2}\left(m_{\tilde{\chi}}\right)=\min _{\vec{p}_{\mathrm{T}}^{\tilde{\chi}(1)}+\vec{p}_{\mathrm{T}}^{\widetilde{\chi}(2)}=\vec{p}_{\mathrm{T}}^{\text {miss }}}\left[\max \left(M_{\mathrm{T}}^{(1)}, M_{\mathrm{T}}^{(2)}\right)\right],
$$

where the LSP mass $m_{\tilde{\chi}}$ remains a free parameter. This formula can be understood as follows. As neither $M_{\mathrm{T}}^{(1)}$ nor $M_{\mathrm{T}}^{(2)}$ can exceed the parent mass if the true momenta are used, the larger of the two can be chosen. To make sure that $M_{\mathrm{T} 2}$ does not exceed the parent mass, a minimization is performed on trial LSP momenta fulfilling the $\vec{p}_{\mathrm{T}}^{\text {miss }}$ constraint. The distribution of $M_{\mathrm{T} 2}$ for the correct value of $m_{\tilde{\chi}}$ then has an endpoint at the value of the primary particle mass. If, however, $m_{\tilde{\chi}}$ is lower (higher) than the correct mass value, the endpoint will be below (above) the parent mass. An analytic expression for $M_{\mathrm{T} 2}$ has been computed [13] assuming that initial-state radiation (ISR) can be neglected. In practice, the determination of $M_{\mathrm{T} 2}$ may be complicated by the presence of ISR or, equivalently, transverse momentum arising from decays that occur upstream in the decay chain [14]. In this case, no analytic expression for $M_{\mathrm{T} 2}$ is known, but it can be computed numerically, using, e.g., the results of ref. [15].

To illustrate the behavior of $M_{\mathrm{T} 2}$, we consider the simple example of $M_{\mathrm{T} 2}$ without ISR or upstream transverse momentum. As discussed in ref. [13], the angular and $p_{\mathrm{T}}$ dependence of $M_{\mathrm{T} 2}$ is encoded in a variable $A_{\mathrm{T}}$ :

$$
A_{\mathrm{T}}=E_{\mathrm{T}}^{\mathrm{vis}(1)} E_{\mathrm{T}}^{\mathrm{vis}(2)}+\vec{p}_{\mathrm{T}}^{\mathrm{vis}(1)} \cdot \vec{p}_{\mathrm{T}}^{\mathrm{vis}(2)}
$$

and $M_{\mathrm{T} 2}$ increases as $A_{\mathrm{T}}$ increases. Therefore, the minimum value of $M_{\mathrm{T} 2}$ is reached in configurations where the visible systems are back-to-back. The maximum value is reached when they are parallel to each other and have large $p_{\mathrm{T}}$. In the simple case where $m_{\tilde{\chi}}=0$ and the visible systems have zero mass, $M_{\mathrm{T} 2}$ becomes

$$
\left(M_{\mathrm{T} 2}\right)^{2}=2 A_{\mathrm{T}}=2 p_{\mathrm{T}}^{\mathrm{vis}(1)} p_{\mathrm{T}}^{\mathrm{vis}(2)}\left(1+\cos \phi_{12}\right),
$$

where $\phi_{12}$ is the angle between the two visible systems in the transverse plane. It can be seen that eq. (2.4) corresponds to the transverse mass of two systems $\left(M_{\mathrm{T}}\right)^{2}=2 p_{\mathrm{T}}^{\mathrm{sys}(1)} p_{\mathrm{T}}^{\mathrm{sys}(2)}(1-$ $\left.\cos \phi_{12}\right)$, with $\vec{p}_{\mathrm{T}}^{\mathrm{vis}}=-\vec{p}_{\mathrm{T}}^{\text {sys }}$ for one of the systems.

In this paper, we use $M_{\mathrm{T} 2}$ as a variable to distinguish potential new physics events from SM backgrounds. The use of $M_{\mathrm{T} 2}$ as a discovery variable was first proposed in ref. [16] , but here we follow a different approach. Several choices for the visible system used as input to $M_{\mathrm{T} 2}$ can be considered: dijet events (as in ref. [16]), the two jets with largest $p_{\mathrm{T}}$ 
in multijet events, or two systems of pseudojets defined by grouping jets together. In this study, we use the last method.

A technique to group jets in multijet events into two pseudojets is the "event hemispheres" method described in ref. [17] (see section 13.4). We take the two initial axes as the directions of the two massless jets that yield the largest dijet invariant mass. The pseudojets are then formed based on a minimization of the Lund distance criterion $[17,18]$.

We use $M_{\mathrm{T} 2}$ as our main search variable since SUSY events with large expected $E_{\mathrm{T}}^{\text {miss }}$ and jet acoplanarity will be concentrated in the large $M_{\mathrm{T} 2}$ region. In contrast, QCD dijet events, in which the two jets are back-to-back, populate the region of small $M_{\mathrm{T} 2}$ regardless of the value of $E_{\mathrm{T}}^{\text {miss }}$ or jet $p_{\mathrm{T}}$. In the present study, we choose the visible systems to be massless and set $m_{\tilde{\chi}}=0$. Then back-to-back dijet events will have $M_{\mathrm{T} 2}=0$, as explained above. Hence, $M_{\mathrm{T} 2}$ has a built-in protection against jet mismeasurements in QCD dijet events, even if accompanied by large $E_{\mathrm{T}}^{\text {miss }}$. However, QCD multijet events with large $E_{\mathrm{T}}^{\text {miss }}$ may give rise to acoplanar pseudojets, leading to larger $M_{\mathrm{T} 2}$ values. For this reason, further protections against $E_{\mathrm{T}}^{\text {miss }}$ from mismeasurements need to be introduced, as described below. Other SM backgrounds, such as t $\bar{t}$, single top-quark, and $\mathrm{W}+$ jets events with leptonic decays, or $\mathrm{Z}+$ jets events where the $\mathrm{Z}$ boson decays to neutrinos, contain true $E_{\mathrm{T}}^{\mathrm{miss}}$ and can also lead to acoplanar pseudojets.

\section{CMS detector}

The central feature of the CMS apparatus is a superconducting solenoid $13 \mathrm{~m}$ in length and $6 \mathrm{~m}$ in diameter that provides an axial magnetic field of $3.8 \mathrm{~T}$. The core of the solenoid is instrumented with various particle detection systems: a silicon pixel and strip tracker, an electromagnetic calorimeter (ECAL), and a brass/scintillator hadron calorimeter (HCAL). The silicon pixel and strip tracker covers $|\eta|<2.5$, where pseudorapidity $\eta$ is defined by $\eta=-\ln [\tan (\theta / 2)]$ with $\theta$ the polar angle of the trajectory of the particle with respect to the counterclockwise beam direction. The ECAL and HCAL cover $|\eta|<3$. The steel return yoke outside the solenoid is instrumented with gas detectors used to identify muons. A quartz-steel Cerenkov-radiation-based forward hadron calorimeter extends the coverage to $|\eta| \leq 5$. The detector is nearly hermetic, covering $0<\phi<2 \pi$ in azimuth, allowing for energy balance measurements in the plane transverse to the beam directions. The first level of the CMS trigger system, composed of custom hardware processors, uses information from the calorimeters and muon detectors to select the most interesting events in a fixed time interval of less than $4 \mu \mathrm{s}$. The High Level Trigger processor farm further decreases the event rate from around $100 \mathrm{kHz}$ to around $300 \mathrm{~Hz}$, before data storage. A detailed description of the CMS detector can be found elsewhere [19].

\section{Samples and event selection}

The data used in this analysis were collected by triggers based on the quantity $H_{\mathrm{T}}$, the scalar sum of transverse momenta of reconstructed and energy-corrected calorimeter jets. Due to a continuous increase in the instantaneous luminosity of the LHC, the trigger 
evolved with time from the requirement $H_{\mathrm{T}}>440 \mathrm{GeV}$ to $H_{\mathrm{T}}>750 \mathrm{GeV}$. In this analysis, only triggers with a threshold of $650 \mathrm{GeV}$ or less have been used, corresponding to a total integrated luminosity of $4.73 \mathrm{fb}^{-1}$.

The analysis is designed using simulated event samples created with the PYTHIA 6.4.22 [18] and MadGraph 5v1.1 [20] Monte Carlo event generators. These events are subsequently processed with a detailed simulation of the CMS detector response based on GEant4 [21]. The events are reconstructed and analyzed in the same way as the data. The SUSY signal particle spectrum is calculated using SOFTSUSY [22] and for the decays SDECAY [23] is used. We use two CMS SUSY benchmark signal samples, referred to as LM6 and LM9 [17], to illustrate possible CMSSM [24] yields. The CMSSM is defined by the universal scalar and gaugino mass parameters $m_{0}$ and $m_{1 / 2}$, respectively, the parameter $A_{0}$ of the trilinear couplings, the ratio of the vacuum expectation values of the two Higgs fields $\tan \beta$, and the sign of the Higgs mixing parameter $\operatorname{sign}(\mu)$. The parameter values for LM6 are $m_{0}=85 \mathrm{GeV}, m_{1 / 2}=400 \mathrm{GeV}, \tan \beta=10, A_{0}=0 \mathrm{GeV}$ and $\operatorname{sign}(\mu)>0$. Those for LM9 are $m_{0}=1450 \mathrm{GeV}, m_{1 / 2}=175 \mathrm{GeV}, \tan \beta=50, A_{0}=0 \mathrm{GeV}$ and $\operatorname{sign}(\mu)>0$. All samples are generated using the CTEQ6 [25] parton distribution functions (PDFs). For SM background simulated samples we use the most accurate calculation of the cross sections currently available, usually with next-to-leading-order (NLO) accuracy. For the CMS SUSY benchmark signal samples we use NLO cross sections of $0.403 \mathrm{pb}$ and $10.6 \mathrm{pb}$ for LM6 and LM9, respectively, obtained by weighting the leading order cross sections from PYTHIA with sub-process dependent K-factors calculated with PROSPINO [26].

The events are reconstructed using the particle-flow (PF) algorithm [27], which identifies and reconstructs individually the particles produced in the collision, namely charged hadrons, photons, neutral hadrons, electrons, and muons.

Electrons and muons with $p_{\mathrm{T}} \geq 10 \mathrm{GeV}$ and $|\eta| \leq 2.4$ are considered isolated if the transverse momentum sum of charged hadrons, photons, and neutral hadrons surrounding the lepton within a cone of radius $\sqrt{(\Delta \eta)^{2}+(\Delta \phi)^{2}}=0.4$, divided by the lepton transverse momentum value itself, is less than 0.2. The electron and muon reconstruction and identification algorithms are described in refs. [28, 29] and [30], respectively. All particles apart from the isolated electrons and muons are clustered into jets using the anti- $k_{\mathrm{T}}$ jet clustering algorithm [31] with distance parameter 0.5 [32, 33]. Jet energies are calibrated by applying correction factors as a function of the transverse momentum and the pseudorapidity of the jet. Residual jet energy corrections are applied to jets in data to account for differences in jet energy scale between simulation and data [34]. The effect of pileup, namely multiple pp collisions within a beam crossing, is reduced by using the FastJet pileup subtraction procedure $[35,36]$ for data and simulated events. Jets are required to pass loose identification criteria and to satisfy $p_{\mathrm{T}}>20 \mathrm{GeV}$ and $|\eta| \leq 2.4$. The b-jet tagging is based on the simple-secondary-vertex algorithm [37]. We use the high-purity working point that yields a typical jet-tagging efficiency of $42 \%$ for b jets in our search region while the mistagging efficiency for light-flavored (uds quark and gluon) jets is of the order of $0.1 \%$ and for c jets, $6.3 \%$. The missing transverse momentum $\vec{E}_{\mathrm{T}}^{\text {miss }}$ is computed as the negative vector sum of all particles reconstructed by the PF algorithm [33].

Events are required to contain at least one good primary vertex [38]. The $H_{\mathrm{T}}$ value, computed from PF jets with $p_{\mathrm{T}}>50 \mathrm{GeV}$, must satisfy $H_{\mathrm{T}} \geq 750 \mathrm{GeV}$. With this $H_{\mathrm{T}}$ 
requirement, the triggers are nearly $100 \%$ efficient. At least three jets are required, where a $p_{\mathrm{T}}$ threshold of $40 \mathrm{GeV}$ is used for jet counting. The two leading jets are required to have $p_{\mathrm{T}}>100 \mathrm{GeV}$. The value of $E_{\mathrm{T}}^{\text {miss }}$ is required to exceed $30 \mathrm{GeV}$. Events containing beam background or anomalous calorimeter noise are rejected. To reject events where a significant fraction of the momentum imbalance arises from forward or soft jets, a maximum difference of $70 \mathrm{GeV}$ is imposed on the modulus of the difference between the $\vec{E}_{\mathrm{T}}^{\text {miss }}$ and $\vec{H}_{\mathrm{T}}^{\text {miss }}$ vectors, where $\vec{H}_{\mathrm{T}}^{\text {miss }}$ is the negative vector sum of all selected jets. Events containing jet candidates with $p_{\mathrm{T}}>50 \mathrm{GeV}$ that fail the jet identification criteria are also rejected.

To reduce the background from QCD multijet events with large $E_{\mathrm{T}}^{\mathrm{miss}}$, arising from mismeasurements or leptonic heavy flavor decays, a minimum azimuthal difference $\Delta \phi_{\min }\left(\right.$ jets, $\left.\vec{E}_{\mathrm{T}}^{\text {miss }}\right)>0.3$ is required between the directions of $\vec{E}_{\mathrm{T}}^{\mathrm{miss}}$ and any jet with $p_{\mathrm{T}}>20 \mathrm{GeV}$. Finally, events are rejected if they contain an isolated electron or muon, to suppress the contributions from $\mathrm{W}+$ jets, $\mathrm{Z}+$ jets and top-quark backgrounds.

\section{Search strategy}

The $M_{\mathrm{T} 2}$ variable is computed after applying the selection criteria of section 4 . We separately consider fully hadronic channels with $\geq 3$ jets and a tight $M_{\mathrm{T} 2}$ requirement (the $M_{\mathrm{T} 2}$ analysis), which is mostly sensitive to signal regions with large squark and gluino masses, and channels with $\geq 4$ jets, at least one tagged $\mathrm{b}$ jet, and a relaxed $M_{\mathrm{T} 2}$ requirement (the $M_{\mathrm{T} 2} b$ analysis), which increases sensitivity to regions with small gluino and large squark masses.

Given the event selection outlined above, we do not expect a significant number of QCD multijet events to appear in the signal regions. Nonetheless, we estimate an upper limit on the remaining QCD multijet background in the signal regions from data control samples. The main backgrounds, consisting of $\mathrm{W}+$ jets, $\mathrm{Z}+$ jets, and top-quark production, are evaluated from data control samples and simulation. A common strategy is applied to both the $M_{\mathrm{T} 2}$ and $M_{\mathrm{T} 2} b$ analyses:

- Two regions are defined in $H_{\mathrm{T}}$, a low $H_{\mathrm{T}}$ region $750 \leq H_{\mathrm{T}}<950 \mathrm{GeV}$ and a high $H_{\mathrm{T}}$ region $H_{\mathrm{T}} \geq 950 \mathrm{GeV}$. In each region, several adjacent bins in $M_{\mathrm{T} 2}$ are defined: five bins for the $M_{\mathrm{T} 2}$ analysis and four for the $M_{\mathrm{T} 2} b$ analysis. The lowest bin in $M_{\mathrm{T} 2}$ is chosen such that the expected QCD multijet background remains a small fraction of the total background. For the $M_{\mathrm{T} 2}$ analysis the lowest bin starts at $M_{\mathrm{T} 2}=150 \mathrm{GeV}$ and for $M_{\mathrm{T} 2} b$ at $M_{\mathrm{T} 2}=125 \mathrm{GeV}$.

- A dedicated method for each background is designed to estimate its contribution in the signal region from data control samples and simulation. The number of events and their relative systematic uncertainties are computed by means of these methods in each $H_{\mathrm{T}}, M_{\mathrm{T} 2}$ bin. The methods are designed such that the resulting estimates are largely uncorrelated statistically.

- The predicted number of events for all background components and their uncertainties are combined, resulting in an estimate of the total background yield and its uncertainty in each bin. 


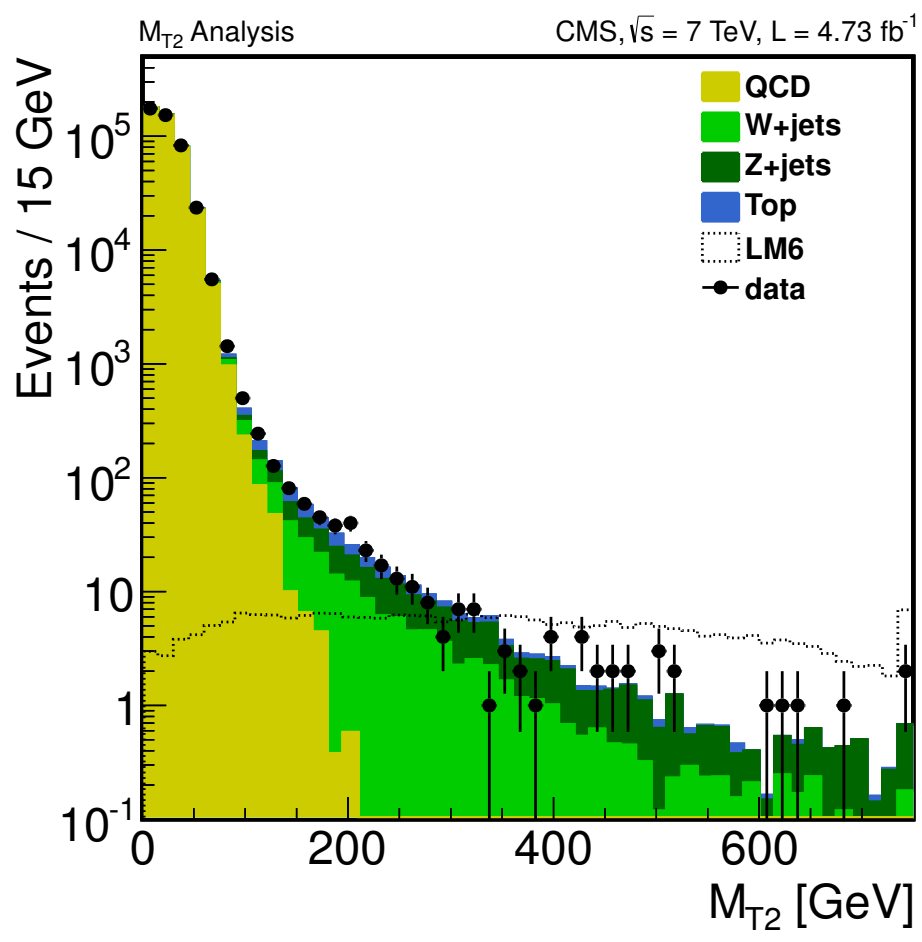

Figure 1. The $M_{\mathrm{T} 2}$ distribution with all selection requirements applied and $H_{\mathrm{T}} \geq 750 \mathrm{GeV}$. The different predictions for the SM backgrounds from simulation are stacked on top of each other. The LM6 signal distribution is not stacked. All distributions from simulation are normalized to the integrated luminosity of the data.

- The estimated number of background events for each bin is compared to the number of observed events, and the potential contribution from a SUSY signal is quantified by a statistical method described in section 8 .

\section{$6 \quad M_{\mathrm{T} 2}$ analysis}

Figure 1 shows the measured $M_{\mathrm{T} 2}$ distribution in comparison to simulation. For $M_{\mathrm{T} 2}<$ $80 \mathrm{GeV}$ the distribution is completely dominated by QCD multijet events. For medium $M_{\mathrm{T} 2}$ values, the distribution is dominated by $\mathrm{W}+$ jets and $\mathrm{Z}(\nu \bar{\nu})+$ jets events with some contribution from top-quark events, while in the tail of $M_{\mathrm{T} 2}$ the contribution from top-quark production becomes negligible and $\mathrm{Z}(\nu \bar{\nu})+$ jets together with $\mathrm{W}+$ jets events dominate. We observe good agreement between data and simulation in the core as well as in the tail of the distribution. The white histogram (black dotted line) corresponds to the LM6 signal. It can be noted that in the presence of signal, an excess in the tail of $M_{\mathrm{T} 2}$ is expected.

The corresponding event yields for data and SM simulated samples, after the full selection and for the various bins in $M_{\mathrm{T} 2}$, are given in table 1 for the low and the high $H_{\mathrm{T}}$ regions. Contributions from other backgrounds, such as $\gamma+$ jets, $Z(\ell \ell)+$ jets and diboson production, are found to be negligible. It is seen that for all but one $M_{\mathrm{T} 2}$ bin, the observed number of events agrees within the uncertainties with the SM background expectation from 


\begin{tabular}{|c|c|c|c|c|c|c|}
\hline & QCD multijet & $W+$ jets & Top & $Z(\nu \nu)+$ jets & Total SM & Data \\
\hline \multicolumn{7}{|c|}{$750 \leq H_{\mathrm{T}}<950$} \\
\hline$M_{\mathrm{T} 2}[0, \infty]$ & $3.18 \mathrm{e}+05$ & $9.22 \mathrm{e}+02$ & $1.30 \mathrm{e}+03$ & $3.01 \mathrm{e}+02$ & $3.20 \mathrm{e}+05$ & $3.20 \mathrm{e}+05$ \\
\hline$M_{\mathrm{T} 2}[150,200]$ & 3.08 & 37.5 & 20.6 & 27.9 & 90.0 & 88 \\
\hline$M_{\mathrm{T} 2}[200,275]$ & 0.0 & 20.6 & 9.40 & 20.3 & 50.3 & 69 \\
\hline$M_{\mathrm{T} 2}[275,375]$ & 0.0 & 9.74 & 2.74 & 11.6 & 24.1 & 19 \\
\hline$M_{\mathrm{T} 2}[375,500]$ & 0.0 & 3.63 & 0.69 & 6.07 & 10.4 & 8 \\
\hline$M_{\mathrm{T} 2}[500, \infty]$ & 0.0 & 1.54 & 0.20 & 3.55 & 5.29 & 6 \\
\hline \multicolumn{7}{|l|}{$H_{\mathrm{T}} \geq 950$} \\
\hline$M_{\mathrm{T} 2}[0, \infty]$ & $1.22 \mathrm{e}+05$ & $4.39 \mathrm{e}+02$ & $6.32 \mathrm{e}+02$ & $1.42 \mathrm{e}+02$ & $1.23 \mathrm{e}+05$ & $1.19 \mathrm{e}+05$ \\
\hline$M_{\mathrm{T} 2}[150,200]$ & 9.84 & 19.8 & 11.7 & 12.9 & 54.2 & 70 \\
\hline$M_{\mathrm{T} 2}[200,275]$ & 0.47 & 13.7 & 5.25 & 10.5 & 30.0 & 23 \\
\hline$M_{\mathrm{T} 2}[275,375]$ & 0.04 & 6.43 & 1.83 & 6.42 & 14.7 & 9 \\
\hline$M_{\mathrm{T} 2}[375,500]$ & 0.0 & 1.63 & 0.40 & 2.54 & 4.57 & 8 \\
\hline$M_{\mathrm{T} 2}[500, \infty]$ & 0.0 & 1.10 & 0.16 & 2.16 & 3.42 & 4 \\
\hline
\end{tabular}

Table 1. Observed number of events and expected SM background yields from simulation in $M_{\mathrm{T} 2}$ bins for the low and high $H_{\mathrm{T}}$ regions. These numbers are for guidance only and are not used in the final background prediction.

simulation. In the low $H_{\mathrm{T}}$ region, the $M_{\mathrm{T} 2}$ bin $[200,275] \mathrm{GeV}$ exhibits an excess in data compared to background. We investigated whether the origin could be instrumental in nature, but did not find evidence for it. It could be of statistical origin. The excess has a marginal impact on the final observed limit. Note that the background numbers in table 1 are for guidance only and are not used in the final background prediction, which will be derived from control regions in the data as described below.

\subsection{Background prediction}

\subsubsection{QCD multijet background}

The simulation predicts that the QCD multijet background is negligible in the tail of the $M_{\mathrm{T} 2}$ distribution. Nevertheless, a dedicated method using a data control region was designed to verify that this is indeed the case.

We base this estimation on $M_{\mathrm{T} 2}$ and $\Delta \phi_{\min }$, which is the difference in azimuth between $\vec{E}_{\mathrm{T}}^{\mathrm{miss}}$ and the closest jet. The background in the signal region, defined by $\Delta \phi_{\min } \geq 0.3$ and large $M_{\mathrm{T} 2}$, is predicted from a control region with $\Delta \phi_{\min } \leq 0.2$. The two variables are strongly correlated, but a factorization method can still be applied if the functional form is known for the ratio of the number of events $r\left(M_{\mathrm{T} 2}\right)=N\left(\Delta \phi_{\min } \geq 0.3\right) / N\left(\Delta \phi_{\min } \leq 0.2\right)$ as a function of $M_{\mathrm{T} 2}$. It is found from simulation studies, and confirmed with data, that 
for $M_{\mathrm{T} 2}>50 \mathrm{GeV}$ the ratio falls exponentially. Therefore, a parameterization of the form

$$
r\left(M_{\mathrm{T} 2}\right)=\frac{N\left(\Delta \phi_{\min } \geq 0.3\right)}{N\left(\Delta \phi_{\min } \leq 0.2\right)}=\exp \left(a-b M_{\mathrm{T} 2}\right)+c
$$

is used for $M_{\mathrm{T} 2}>50 \mathrm{GeV}$. The function is assumed to reach a constant value at large $M_{\mathrm{T} 2}$ due to extreme tails of the jet energy resolution response.

The method is validated with simulation. First the parameters $a, b$, and $c$ are extracted from a fit to simulated QCD multijet events in the full $M_{\mathrm{T} 2}$ spectrum. The fitted parameter value for $c$ is compatible with a negligible QCD multijet contribution at large $M_{\mathrm{T} 2}$. It is verified that similar fit results for the parameters $a$ and $b$ are obtained when the fit is limited to the region $50<M_{\mathrm{T} 2}<80 \mathrm{GeV}$, where contributions from background processes other than that from QCD multijets is small. The robustness of the prediction is checked by systematically varying the fit boundaries.

For the final results, we repeat the fit to data in the region $50<M_{\mathrm{T} 2}<80 \mathrm{GeV}$, after subtracting the $\mathrm{W}+$ jets, $\mathrm{Z}+$ jets and top background contributions using simulation. The fitted parameter values for $a$ and $b$ are in agreement with the values obtained from the QCD multijet simulation. We conservatively fix the constant $c$ to the value of the exponential at $M_{\mathrm{T} 2}=250 \mathrm{GeV}$, where agreement with data can still be verified. In the lower $M_{\mathrm{T} 2}$ bins, where the exponential term dominates, the method reliably predicts the QCD multijet background. For higher $M_{\mathrm{T} 2}$ bins, where the constant term dominates, the method overestimates the number of QCD multijet events relative to the simulation, nonetheless confirming that the QCD multijet contribution is negligible.

The extreme case of total loss of a jet, leading to population of the high $M_{\mathrm{T} 2}$ tail, is studied using a sample of high $p_{\mathrm{T}}$ mono-jet events obtained with a dedicated event selection. The total number of events is found to be compatible within the uncertainties with the number expected from the electroweak processes, confirming that the QCD multijet contribution is negligible and hence that the constant $c$ is small.

\subsection{2 $\mathrm{W}(\ell \nu)+$ jets and top-quark background}

The backgrounds due to $\mathrm{W}(\ell \nu)+$ jets and to semi-leptonic decays of top quarks have the following sources in common:

- leptonic decays of the $\mathrm{W}$ boson, where the lepton is unobserved because it falls outside the $p_{\mathrm{T}}$ or $\eta$ acceptance;

- to a lesser extent, leptonic decays of the $\mathrm{W}$ boson, where the lepton is within the acceptance but fails to satisfy the reconstruction, identification, or isolation criteria;

- $\mathrm{W}\left(\tau \nu_{\tau}\right)$ decays, where the $\tau$ decays hadronically.

We refer to leptons that fall into either of the first two categories as "lost leptons". The number of events with lost leptons is estimated from a data control sample where a single lepton (e or $\mu$ ) is found. A correction factor accounting for the probability to lose the lepton is derived from simulation. To avoid a potential contamination from signal events, a transverse mass cut $M_{\mathrm{T}}<100 \mathrm{GeV}$ is introduced. This method is applied in the various $H_{\mathrm{T}}$ 
and $M_{\mathrm{T} 2}$ bins. First, a successful validation test of the method is performed using simulated samples. Then, a prediction is made from the data bin by bin and found to be in agreement with the expectation from simulation. A systematic uncertainty is evaluated that includes the uncertainty on the lepton efficiencies, acceptance, and background subtraction.

For the background contribution from hadronically decaying tau leptons, a method similar to the one described above is used. Events with an isolated and identified hadronically decaying tau [39] lepton are selected in the various $H_{\mathrm{T}}$ and $M_{\mathrm{T} 2}$ bins. The contribution from jets misidentified as taus is subtracted. The remaining number of tau events is corrected for the tau reconstruction and identification efficiency. The predicted number of hadronically decaying tau background events agrees with the true number from simulation. Given the small number of events in the data, the numbers of events from the simulation are used for the background estimate, with the same relative systematic uncertainties as for the lost leptons.

\subsection{3 $\mathrm{Z}(\nu \bar{\nu})+$ jets background}

The estimate of the $\mathrm{Z}(\nu \bar{\nu})+$ jets background is obtained independently from two distinct data samples, one containing $\gamma+$ jets events and the other $\mathrm{W}(\mu \nu)+$ jets events. In both cases the invisible decay of the $\mathrm{Z}$ boson is mimicked by removing, respectively, the photon and the muon from the event, and adding vectorially the corresponding $\vec{p}_{\mathrm{T}}$ to $\vec{E}_{\mathrm{T}}^{\text {miss }}$.

For the estimate based on $\gamma+$ jets events, a sample of events with identified and isolated photons [40] with $p_{\mathrm{T}}>20 \mathrm{GeV}$ is selected, where all selection requirements except that on $M_{\mathrm{T} 2}$ are imposed. This sample contains both prompt photons and photons from $\pi^{0}$ decays in QCD multijet events. The two components are separated by performing a maximum likelihood fit of templates from simulated events to the shower shapes. The event sample is dominated by low $p_{\mathrm{T}}$ photons, where the shower shape provides high discrimination power between prompt photons and $\pi^{0} \mathrm{~s}$. The extrapolation of their contributions as a function of $M_{\mathrm{T} 2}$ is obtained from simulation. The $\mathrm{Z}(\nu \bar{\nu})+$ jets background is estimated for each bin in $M_{\mathrm{T} 2}$ from the number of prompt photon events multiplied by the $M_{\mathrm{T} 2}$-dependent ratio of $\mathrm{Z}(\nu \bar{\nu})+$ jets to $\gamma+$ jets events obtained from simulation. This ratio increases as a function of the photon $p_{\mathrm{T}}$ (which drives the $M_{\mathrm{T} 2}$ value) and reaches a constant value above $300 \mathrm{GeV}$. The resulting prediction of the background is found to be in good agreement with the expectation from simulation. Systematic uncertainties on the background prediction consist of the statistical uncertainties from the number of $\gamma+$ jets events, a normalization uncertainty in the shower shape fit of $5 \%$, and the systematic uncertainties on the ratio of $\mathrm{Z}(\nu \bar{\nu})+$ jets to $\gamma+$ jets events in the simulation. The uncertainties on the ratio are estimated to be less than $20 \%(30 \%)$ for $M_{\mathrm{T} 2}<275\left(M_{\mathrm{T} 2}>275\right) \mathrm{GeV}$. To assess these uncertainties, the $p_{\mathrm{T}}$ dependence of the ratio is studied in data and compared to simulation using leptonically decaying $\mathrm{Z}$ events. For $p_{\mathrm{T}}>400 \mathrm{GeV}$ this test is limited by the number of the leptonic $Z$ events, which justifies the increased uncertainty for $M_{\mathrm{T} 2}>275 \mathrm{GeV}$.

For the estimate from $\mathrm{W}(\mu \nu)+$ jets events, corrections are needed for lepton acceptance, lepton reconstruction efficiency, and the ratio between the production cross sections for $\mathrm{W}$ and $\mathrm{Z}$ bosons (including differences between the shapes of the distributions on which selection criteria are applied). The lepton efficiencies are taken from studies of $\mathrm{Z}(\mu \mu)$ events in 


\begin{tabular}{|c|c|c|c|c|c|c|c|c|c|}
\hline & sim. & $\begin{array}{l}\rightarrow \nu \bar{\nu} \\
\text { data pred. }\end{array}$ & $\begin{array}{r}\mathrm{Lc} \\
\operatorname{sim} .\end{array}$ & $\begin{array}{l}\text { st lepton } \\
\text { data pred. }\end{array}$ & $\begin{array}{l}\tau \rightarrow \text { had } \\
\text { Estimate }\end{array}$ & $\begin{array}{r}\mathrm{QC} \\
\text { sim. }\end{array}$ & $\begin{array}{l}\text { D multijet } \\
\text { data pred. }\end{array}$ & $\begin{array}{l}\text { Total bkg. } \\
\text { data pred. }\end{array}$ & Data \\
\hline \multicolumn{10}{|c|}{$750 \leq H_{\mathrm{T}}<950$} \\
\hline$M_{\mathrm{T} 2}[150,200]$ & 27.9 & $24.2 \pm 4.9$ & 36.0 & $29.6 \pm 7.1$ & $22.5 \pm 5.4$ & 3.1 & $7.0 \pm 3.5$ & $83.3 \pm 10.7$ & 88 \\
\hline$M_{\mathrm{T} 2}[200,275]$ & 20.3 & $21.8 \pm 4.8$ & 17.2 & $11.9 \pm 3.9$ & $12.7 \pm 4.2$ & 0.0 & $1.0 \pm 0.5$ & $47.4 \pm 7.5$ & 69 \\
\hline$M_{\mathrm{T} 2}[275,375]$ & 11.6 & $13.7 \pm 3.8$ & 7.1 & $4.2 \pm 1.9$ & $5.4 \pm 2.5$ & 0.0 & $0.14 \pm 0.07$ & $23.4 \pm 4.9$ & 19 \\
\hline$M_{\mathrm{T} 2}[375,500]$ & 6.1 & $4.1 \pm 1.6$ & 2.2 & $1.1 \pm 0.9$ & $2.2 \pm 1.8$ & 0.0 & $0.08 \pm 0.05$ & $7.4 \pm 2.6$ & 8 \\
\hline$M_{\mathrm{T} 2}[500, \infty]$ & 3.5 & $1.8 \pm 0.9$ & 1.1 & $1.2 \pm 1.0$ & $0.6 \pm 0.5$ & 0.0 & $0.00 \pm 0.00$ & $3.6 \pm 1.4$ & 6 \\
\hline \multicolumn{10}{|l|}{$H_{\mathrm{T}} \geq 950$} \\
\hline$M_{\mathrm{T} 2}[150,200]$ & 12.9 & $16.7 \pm 3.6$ & 18.7 & $16.2 \pm 5.3$ & $12.7 \pm 4.1$ & 9.8 & $11.0 \pm 5.5$ & $56.6 \pm 9.4$ & 70 \\
\hline$M_{\mathrm{T} 2}[200,275]$ & 10.5 & $4.5 \pm 2.0$ & 11.7 & $10.2 \pm 3.7$ & $7.1 \pm 2.6$ & 0.47 & $1.4 \pm 0.7$ & $23.2 \pm 5.0$ & 23 \\
\hline$M_{\mathrm{T} 2}[275,375]$ & 6.4 & $5.7 \pm 2.2$ & 5.0 & $2.9 \pm 1.7$ & $3.3 \pm 1.9$ & 0.04 & $0.13 \pm 0.07$ & $12.1 \pm 3.3$ & 9 \\
\hline$M_{\mathrm{T} 2}[375,500]$ & 2.5 & $3.0 \pm 1.4$ & 1.1 & $0.6 \pm 0.6$ & $0.9 \pm 0.9$ & 0.0 & $0.06 \pm 0.04$ & $4.6 \pm 1.8$ & 8 \\
\hline$M_{\mathrm{T} 2}[500, \infty]$ & 2.2 & $2.5 \pm 1.5$ & 0.6 & $0.6 \pm 0.6$ & $0.6 \pm 0.6$ & 0.0 & $0.06 \pm 0.04$ & $3.8 \pm 1.7$ & 4 \\
\hline
\end{tabular}

Table 2. Estimated event yields for each background contribution in the various $M_{\mathrm{T} 2}$ and $H_{\mathrm{T}}$ bins. The predictions from control regions in data are compared to the expected event yields from simulation. Statistical and systematic uncertainties are added in quadrature. The total background prediction is compared to data in the last two columns.

data. Also, the top-quark background to the $\mathrm{W}+$ jets sample is subtracted. The top-quark background is evaluated by applying $\mathrm{b}$ tagging to the data to identify top-quark decays and then correcting for the b-tagging efficiency. The $\mathrm{Z}(\nu \bar{\nu})+$ jets background is then estimated in each of the $M_{\mathrm{T} 2}$ bins. The systematic uncertainty includes the contributions from the lepton selection and reconstruction efficiencies, the b-tagging efficiency, the acceptance from simulation, and the $\mathrm{W}$-to- $\mathrm{Z}$ ratio.

The $\mathrm{Z}(\nu \bar{\nu})+$ jets background estimates from the $\gamma+$ jets and $\mathrm{W}(\mu \nu)+$ jets methods are in good agreement with each other. Since they are statistically uncorrelated, we take the weighted average of the two predictions as the final estimate.

\subsection{Results}

The results of the background estimation methods for each background contribution are summarized in table 2 and shown in figure 2.

\section{$7 \quad M_{\mathrm{T} 2} b$ analysis}

The selection criteria developed for the $M_{\mathrm{T} 2}$ analysis are not optimal for events with heavy squarks and light gluinos, such as are predicted by the SUSY benchmark model LM9. To improve sensitivity to these types of events, we perform the $M_{\mathrm{T} 2} b$ analysis based on loosened kinematic selection criteria and the requirement of a tagged $\mathrm{b}$ jet. The restriction on $M_{\mathrm{T} 2}$ is loosened to $M_{\mathrm{T} 2}>125 \mathrm{GeV}$ and the $\Delta \phi_{\min }\left(\right.$ jets, $\left.\vec{E}_{\mathrm{T}}^{\text {miss }}\right)>0.3$ requirement is applied to the four leading jets only. We require that there be at least four jets with $p_{\mathrm{T}}>40 \mathrm{GeV}$, and the leading jet to have $p_{\mathrm{T}}>150 \mathrm{GeV}$. We further require that at least one of the jets in the event be tagged as a b-quark jet. 

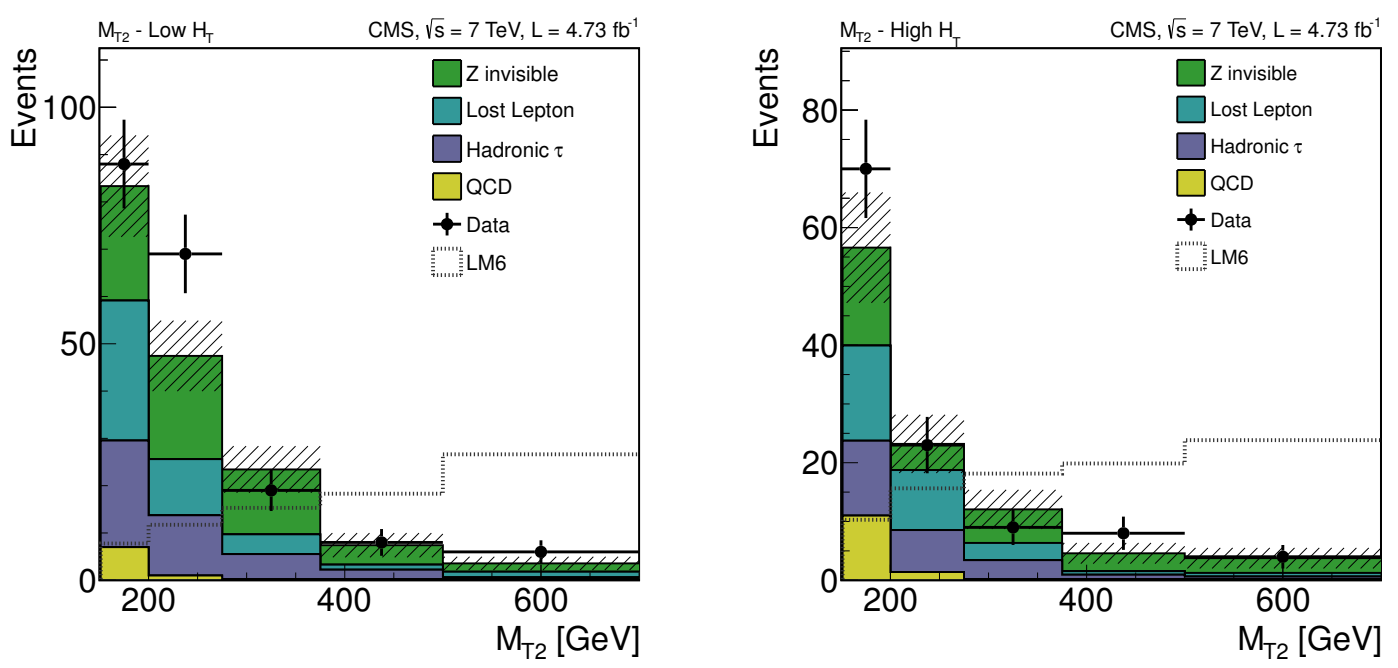

Figure 2. $M_{\mathrm{T} 2}$ distribution from the background estimates compared to data. The figure on the left corresponds to the $750 \leq H_{\mathrm{T}}<950 \mathrm{GeV}$ region, while that on the right corresponds to $H_{\mathrm{T}} \geq 950 \mathrm{GeV}$. The predictions from simulated events for the LM6 signal model (not stacked) are also shown. The hatched band shows the total uncertainty on the SM background estimate.

Figure 3 shows the $M_{\mathrm{T} 2}$ distribution for events that satisfy the $M_{\mathrm{T} 2} b$ selection criteria and with $H_{\mathrm{T}} \geq 750 \mathrm{GeV}$. As for the $M_{\mathrm{T} 2}$ analysis (figure 1), the QCD multijet background dominates for $M_{\mathrm{T} 2}<80 \mathrm{GeV}$ but is strongly suppressed for $M_{\mathrm{T} 2} \geq 125 \mathrm{GeV}$. In the signal region, top-quark events dominate the electroweak contribution. The white histogram (black dotted line) corresponds to the LM9 signal. The corresponding event yields for data and SM simulation for the low and high $H_{\mathrm{T}}$ regions are summarized in table 3 . Note that the background numbers in table 3 are for guidance only and are not used in the final background prediction, which will be derived from control regions in the data as described below.

\subsection{Background prediction and results}

The QCD multijet contribution is estimated following the same approach as for the $M_{\mathrm{T} 2}$ analysis. We find that the function in eq. (6.1) fitted to data in the region $50<M_{\mathrm{T} 2}<80 \mathrm{GeV}$ provides a good description of the QCD multijet background, also for events containing b-tagged jets. From the fit to data, the prediction of the QCD multijet background is obtained in the various $M_{\mathrm{T} 2}$ bins for the low and high $H_{\mathrm{T}}$ regions.

Events arising from top-quark production are the dominant background contribution in the signal region. The top-quark contribution is evaluated, together with the one from $\mathrm{W}(\ell \nu)+$ jets, in the same way as for the $M_{\mathrm{T} 2}$ analysis, using single-electron and single-muon events, as well as taus decaying to hadrons.

The background from $\mathrm{Z}(\nu \bar{\nu})+$ jets events is expected to be very small compared with the background from top-quark events. We estimate the background from $\mathrm{Z}(\nu \bar{\nu})+$ jets events with the method based on $\mathrm{W}+$ jets events discussed for the $M_{\mathrm{T} 2}$ analysis. As the selection of $\mathrm{W}(\mu \nu)+$ jets events includes a b-tag veto to suppress the top-quark background, 


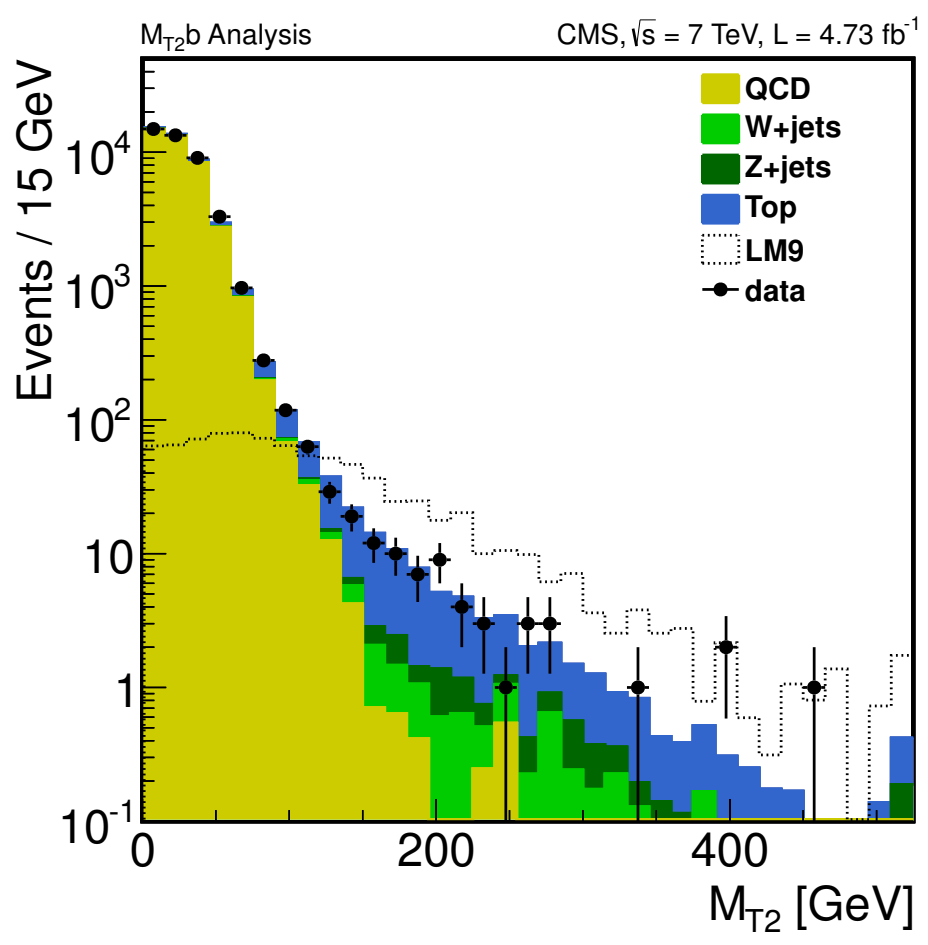

Figure 3. $\quad M_{\mathrm{T} 2}$ for events with the $M_{\mathrm{T} 2} b$ selection criteria applied and with $H_{\mathrm{T}} \geq 750 \mathrm{GeV}$. The different predictions from simulation for the SM backgrounds are stacked on top of each other. The LM9 signal distribution is not stacked. All distributions from simulation are normalized to the integrated luminosity of the data.

a ratio of efficiencies for $\mathrm{W}(\mu \nu)+$ jets events with a b tag to $\mathrm{W}(\mu \nu)+$ jets events without a $\mathrm{b}$ tag is taken into account. This ratio is obtained from simulation.

The results of the estimates for the various backgrounds are summarized in table 4 and shown in figure 4 .

\section{Statistical interpretation of the results and exclusion limits}

No significant deviation from the SM background prediction is observed and upper limits are set on a potential signal. The statistical approach used to derive limits follows closely the methodology of ref. [41]. A brief description of the steps relevant to this analysis follows.

First, a likelihood function is constructed as the product of Poisson probabilities for each $H_{\mathrm{T}}, M_{\mathrm{T} 2}$ search bin. These probabilities are functions of the predicted signal and background yields in each bin. Systematic uncertainties are introduced as nuisance parameters in the signal and background models. Log-normal distributions are taken as a suitable choice for the probability density distributions for the nuisance parameters.

In order to compare the compatibility of the data with the background-only and the signal-plus-background hypotheses, we construct the test statistic $q_{\lambda}$ based on the profile 


\begin{tabular}{|c|c|c|c|c|c|c|}
\hline & QCD multijet & $W+$ jets & Top & $Z(\nu \nu)+$ jets & Total SM & Data \\
\hline \multicolumn{7}{|c|}{$750 \leq H_{\mathrm{T}}<950$} \\
\hline$M_{\mathrm{T} 2}[0, \infty]$ & $2.83 \mathrm{e}+04$ & $4.53 \mathrm{e}+02$ & $1.15 \mathrm{e}+03$ & $1.41 \mathrm{e}+02$ & $2.97 \mathrm{e}+04$ & $2.99 \mathrm{e}+04$ \\
\hline$M_{\mathrm{T} 2}[125,150]$ & 5.16 & 1.86 & 20.3 & 0.95 & 28.3 & 22 \\
\hline$M_{\mathrm{T} 2}[150,200]$ & 0.16 & 1.94 & 17.9 & 2.00 & 22.1 & 16 \\
\hline$M_{\mathrm{T} 2}[200,300]$ & 0.0 & 1.84 & 9.43 & 1.25 & 12.6 & 16 \\
\hline$M_{\mathrm{T} 2}[300, \infty]$ & 0.0 & 0.57 & 2.55 & 0.53 & 3.65 & 2 \\
\hline \multicolumn{7}{|l|}{$H_{\mathrm{T}} \geq 950$} \\
\hline$M_{\mathrm{T} 2}[0, \infty]$ & $1.19 \mathrm{e}+04$ & $2.18 \mathrm{e}+01$ & $5.46 \mathrm{e}+02$ & $6.51 \mathrm{e}+00$ & $1.25 \mathrm{e}+04$ & $1.23 \mathrm{e}+04$ \\
\hline$M_{\mathrm{T} 2}[125,150]$ & 1.25 & 0.76 & 9.95 & 0.64 & 12.7 & 10 \\
\hline$M_{\mathrm{T} 2}[150,180]$ & 0.57 & 0.79 & 7.15 & 0.43 & 8.96 & 10 \\
\hline$M_{\mathrm{T} 2}[180,260]$ & 0.67 & 1.09 & 6.62 & 0.68 & 9.06 & 9 \\
\hline$M_{\mathrm{T} 2}[260, \infty]$ & 0.04 & 0.76 & 3.09 & 0.65 & 4.55 & 3 \\
\hline
\end{tabular}

Table 3. Observed number of events and expected SM background event yields from simulation in the various $M_{\mathrm{T} 2}$ bins for the $M_{\mathrm{T} 2} b$ event selection. These numbers are for guidance only and are not used in the final background prediction.

\begin{tabular}{|c|c|c|c|c|c|c|c|c|c|}
\hline & sim. & $\begin{array}{l}\rightarrow \nu \bar{\nu} \\
\text { data pred. }\end{array}$ & $\begin{array}{r}\text { Lo } \\
\text { sim. }\end{array}$ & $\begin{array}{l}\text { st lepton } \\
\text { data pred. }\end{array}$ & $\begin{array}{l}\tau \rightarrow \text { had } \\
\text { Estimate }\end{array}$ & $\begin{array}{r}\mathrm{QC} \\
\text { sim. }\end{array}$ & $\begin{array}{l}\text { D multijet } \\
\text { data pred. }\end{array}$ & $\begin{array}{l}\text { Total bkg. } \\
\text { data pred. }\end{array}$ & Data \\
\hline \multicolumn{10}{|c|}{$750 \leq H_{\mathrm{T}}<950$} \\
\hline$M_{\mathrm{T} 2}[125,150]$ & 1.0 & $0.5 \pm 0.4$ & 12.8 & $4.5 \pm 3.2$ & $8.7 \pm 6.3$ & 5.16 & $4.1 \pm 2.1$ & $17.8 \pm 7.3$ & 22 \\
\hline$M_{\mathrm{T} 2}[150,200]$ & 2.0 & $0.7 \pm 0.3$ & 11.3 & $7.6 \pm 3.6$ & $8.0 \pm 3.8$ & 0.16 & $0.90 \pm 0.51$ & $17.2 \pm 5.2$ & 16 \\
\hline$M_{\mathrm{T} 2}[200,300]$ & 1.3 & $1.0 \pm 0.5$ & 6.1 & $1.3 \pm 1.7$ & $4.9 \pm 6.7$ & 0.0 & $0.04 \pm 0.03$ & $7.2 \pm 6.9$ & 16 \\
\hline$M_{\mathrm{T} 2}[300, \infty]$ & 0.5 & $0.6 \pm 0.3$ & 1.3 & $1.3 \pm 0.9$ & $1.8 \pm 1.3$ & 0.0 & $0.00 \pm 0.00$ & $3.7 \pm 1.6$ & 2 \\
\hline \multicolumn{10}{|l|}{$H_{\mathrm{T}} \geq 950$} \\
\hline$M_{\mathrm{T} 2}[125,150]$ & 0.6 & $0.4 \pm 0.3$ & 6.2 & $5.9 \pm 3.3$ & $4.3 \pm 2.4$ & 1.25 & $5.4 \pm 2.8$ & $16.0 \pm 4.9$ & 10 \\
\hline$M_{\mathrm{T} 2}[150,180]$ & 0.4 & $0.9 \pm 0.4$ & 4.6 & $6.4 \pm 3.3$ & $3.2 \pm 1.7$ & 0.57 & $1.7 \pm 0.9$ & $12.2 \pm 3.9$ & 10 \\
\hline$M_{\mathrm{T} 2}[180,260]$ & 0.6 & $0.1 \pm 0.1$ & 4.2 & $3.4 \pm 2.3$ & $3.3 \pm 2.3$ & 0.67 & $0.45 \pm 0.25$ & $7.2 \pm 3.2$ & 9 \\
\hline$M_{\mathrm{T} 2}[260, \infty]$ & 0.6 & $0.7 \pm 0.4$ & 2.2 & $2.0 \pm 1.6$ & $1.6 \pm 1.3$ & 0.04 & $0.05 \pm 0.04$ & $4.3 \pm 2.0$ & 3 \\
\hline
\end{tabular}

Table 4. Estimated event yields for each background contribution in the various $M_{\mathrm{T} 2}$ and $H_{\mathrm{T}}$ bins. The predictions from control regions in data are compared to the expected event yields from simulation. Statistical and systematic uncertainties are added in quadrature. The total background prediction is compared to data in the last two columns.

likelihood ratio:

$$
q_{\lambda}=-2 \ln \frac{\mathcal{L}\left(\text { data } \mid \lambda, \hat{\theta}_{\lambda}\right)}{\mathcal{L}(\text { data } \mid \hat{\lambda}, \hat{\theta})}, \quad \text { with } 0 \leq \hat{\lambda} \leq \lambda,
$$

where the signal strength modifier $\lambda$ is introduced to test signal cross section values $\sigma=$ $\lambda \sigma_{\text {sig. }}$. Both the denominator and the numerator are maximized. In the numerator, the signal parameter strength $\lambda$ remains fixed and the likelihood is maximized only for the 

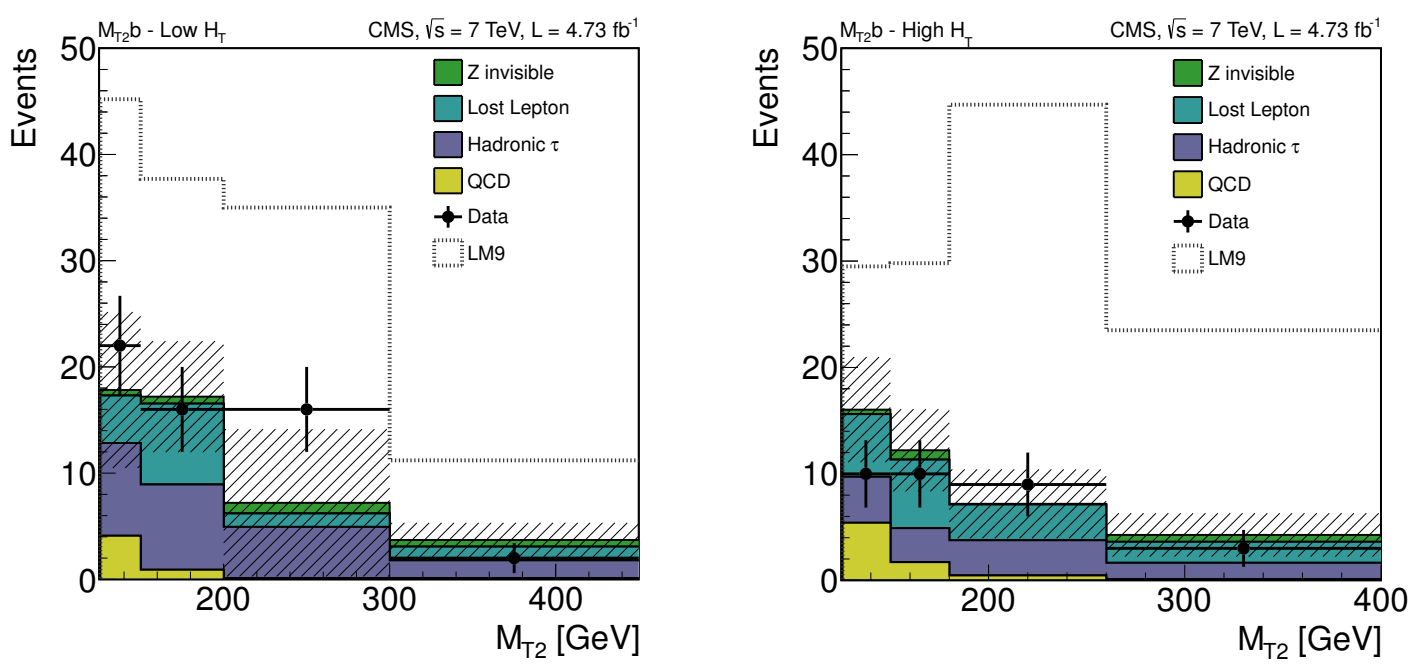

Figure 4. $M_{\mathrm{T} 2}$ distribution from the background estimates compared to data for the $M_{\mathrm{T} 2} b$ selection. The figure on the left corresponds to the $750 \leq H_{\mathrm{T}}<950 \mathrm{GeV}$ region, while that on the right corresponds to $H_{\mathrm{T}} \geq 950 \mathrm{GeV}$. The prediction from simulation for the LM9 signal model (not stacked) are also shown. The hatched band shows the total uncertainty on the SM background estimate.

nuisance parameters, whose values at the maximum are denoted $\hat{\theta}_{\lambda}$. In the denominator, the likelihood is maximized for both $\lambda$ and $\theta . \hat{\lambda}$ and $\hat{\theta}$ denote the values at which $\mathcal{L}$ reaches its global maximum in the denominator. The lower constraint $0 \leq \hat{\lambda}$ is imposed because the signal strength cannot be negative, while the upper constraint $\hat{\lambda}<\lambda$ guarantees a one-sided confidence interval. The value of the test statistic for the actual observation is denoted $q_{\lambda}^{\text {obs }}$. This test statistic [41] differs from that used at LEP and the Tevatron.

To set limits, a modified frequentist $\mathrm{CL}_{\mathrm{s}}$ approach is employed [42, 43]. We first define the probabilities to obtain an outcome of an experiment at least as signal-like as the one observed for the background-only and for the signal-plus-background hypotheses. The $\mathrm{CL}_{\mathrm{s}}$ quantity is then defined as the ratio of these two probabilities. In the modified frequentist approach, the value of $\mathrm{CL}_{\mathrm{s}}$ is required to be less than or equal to $\alpha$ in order to establish a $(1-\alpha)$ confidence level (CL) exclusion. To quote the upper limit on $\lambda$ for a given signal at $95 \% \mathrm{CL}$, we adjust $\lambda$ until we reach $\mathrm{CL}_{\mathrm{s}}=0.05$.

\subsection{Exclusion limits in the CMSSM plane}

Exclusion limits at 95\% CL are determined in the CMSSM $\left(m_{0}, m_{1 / 2}\right)$ plane [44]. The signal cross section is calculated at NLO and next-to-leading-log (NLL) accuracy [26, 45, 46]. At each point in the scan four $\mathrm{CL}_{\mathrm{s}}$ values are computed for $\lambda=1$ : the observed, the median expected, and the one standard deviation $( \pm 1 \sigma)$ expected bands. If the corresponding $\mathrm{CL}_{\mathrm{S}}$ value is smaller than 0.05 , the point is excluded at $95 \% \mathrm{CL}$, resulting in the exclusion limits shown in figure 5 . The results from both the $M_{\mathrm{T} 2}$ and $M_{\mathrm{T} 2} b$ selections are shown in figure 5 (top). In figure 5 (bottom), the results are combined into a single limit exclusion curve based on the best expected limit at each point of the plane. 

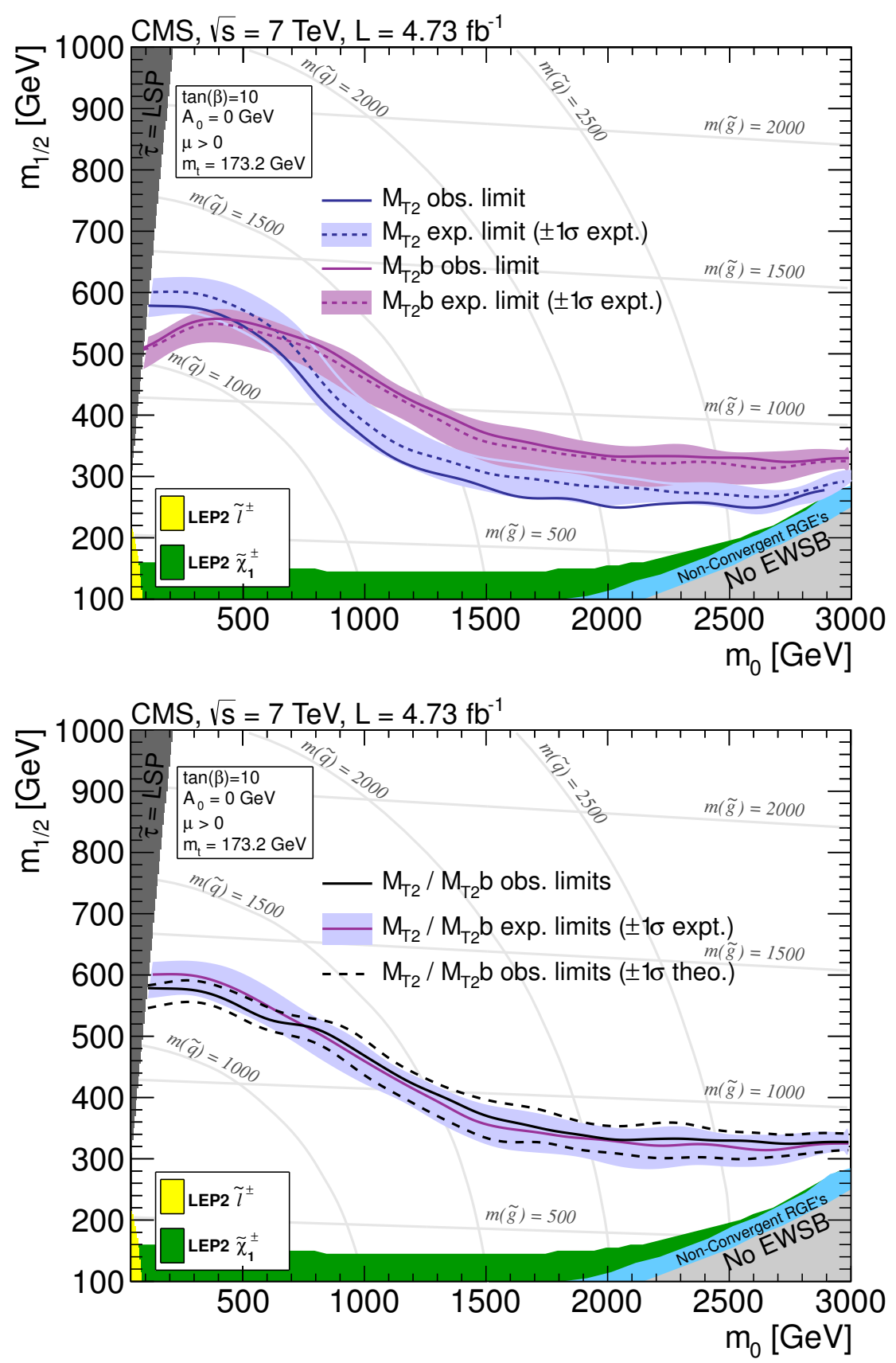

Figure 5. Top: exclusion limit in the CMSSM $\left(m_{0}, m_{1 / 2}\right)$ plane for the $M_{\mathrm{T} 2}$ and $M_{\mathrm{T} 2} b$ analyses with $\tan \beta=10$. Bottom: Combined limit based on the best expected limit at each point.

The dominant sources of systematic uncertainties on the signal model are found to be the jet energy scale and (for the $M_{\mathrm{T} 2} b$ analysis) the b-tagging efficiency. These two uncertainties are evaluated at each point of the CMSSM plane, typically ranging from 5 to $25 \%$ for the former and from 2 to $6 \%$ for the latter. Additionaly, a $2.2 \%$ uncertainty is associated with the luminosity determination [47]. All these uncertainties are included in the statistical interpretation as nuisance parameters on the signal model. 
Observed exclusion limits are also determined when the signal cross section is varied by changing the renormalization and factorization scales by a factor of 2 and using the PDF4LHC recommendation [48] for the PDF uncertainty. The exclusion contours obtained from this method are shown by the dashed curves of figure 5 and referred to as theory uncertainties.

The effect of signal contamination in the leptonic control region could be significant, yielding a potential background overprediction of about 1-15\%. To account for this effect, the signal yields are corrected by subtracting the expected increase in the background estimate that would occur if the given signal were present in the data.

The results in figure 5 (top) establish that the $M_{\mathrm{T} 2}$ analysis is powerful in the region of large squark and gluino masses, corresponding to small $m_{0}$ and large $m_{1 / 2}$, while the $M_{\mathrm{T} 2} b$ analysis increases sensitivity to large squark and small gluino masses, corresponding to large $m_{0}$ and small $m_{1 / 2}$. Conservatively, using the minus one standard deviation $(-1 \sigma)$ theory uncertainty values of the observed limit, we derive absolute lower limits on the squark and gluino masses for the chosen CMSSM parameter set. We find lower limits of $m(\widetilde{\mathrm{q}})>1110 \mathrm{GeV}$ and $m(\widetilde{\mathrm{g}})>800 \mathrm{GeV}$, as well as $m(\widetilde{\mathrm{q}})=m(\widetilde{\mathrm{g}})>1180 \mathrm{GeV}$ assuming equal squark and gluino masses.

\subsection{Exclusion limits for simplified model spectra}

In this section we interpret the results in terms of simplified model spectra [49], which allow a presentation of the exclusion potential in the context of a larger variety of fundamental models, not necessarily in a supersymmetric framework. We studied the following topologies:

- gluino pair production, with $\widetilde{\mathrm{g}} \rightarrow \mathrm{q} \overline{\mathrm{q}} \widetilde{\chi}^{0}$;

- gluino pair production, with $\widetilde{\mathrm{g}} \rightarrow \mathrm{b} \overline{\mathrm{b}} \widetilde{\chi}^{0}$;

- gluino pair production, with $\widetilde{\mathrm{g}} \rightarrow \mathrm{t} \overline{\mathrm{t}} \widetilde{\chi}^{0}$;

- gluino pair production, with $\widetilde{\mathrm{g}} \rightarrow \mathrm{q} \overline{\mathrm{q}} \mathrm{Z} \widetilde{\chi}^{0}$.

The last of these models is used to demonstrate the sensitivity of the analysis in a high jet multiplicity topology, since the hadronic decay of the $\mathrm{Z}$ boson can lead to (maximally) 8 jets in the final state. In figure 6 the 95\% CL excluded cross sections are reported as a function of the relevant masses for gluino pair production with $\widetilde{\mathrm{g}} \rightarrow \mathrm{q} \overline{\mathrm{q}} \widetilde{\chi}^{0}$ using the $M_{\mathrm{T} 2}$ analysis, and for $\widetilde{\mathrm{g}} \rightarrow \mathrm{b} \overline{\mathrm{b}} \widetilde{\chi}^{0}, \widetilde{\mathrm{g}} \rightarrow \mathrm{t} \overline{\mathrm{t}} \widetilde{\chi}^{0}$ and $\widetilde{\mathrm{g}} \rightarrow \mathrm{q} \overline{\mathrm{q}} \widetilde{\chi}^{0}$ using the $M_{\mathrm{T} 2} b$ analysis. Systematic uncertainties on jet energy scale and on b-tagging efficiencies are taken into account as nuisance parameters on the signal model. To minimize the effect of ISR modeling uncertainties, the region near the diagonal is excluded in the limit setting. Observed, median expected, and one standard deviation $( \pm 1 \sigma$ experimental) expected limit curves are derived for the nominal signal cross section. Also shown are the $\pm 1 \sigma$ variation in the observed limit when the signal cross section is varied by its theoretical uncertainties. 

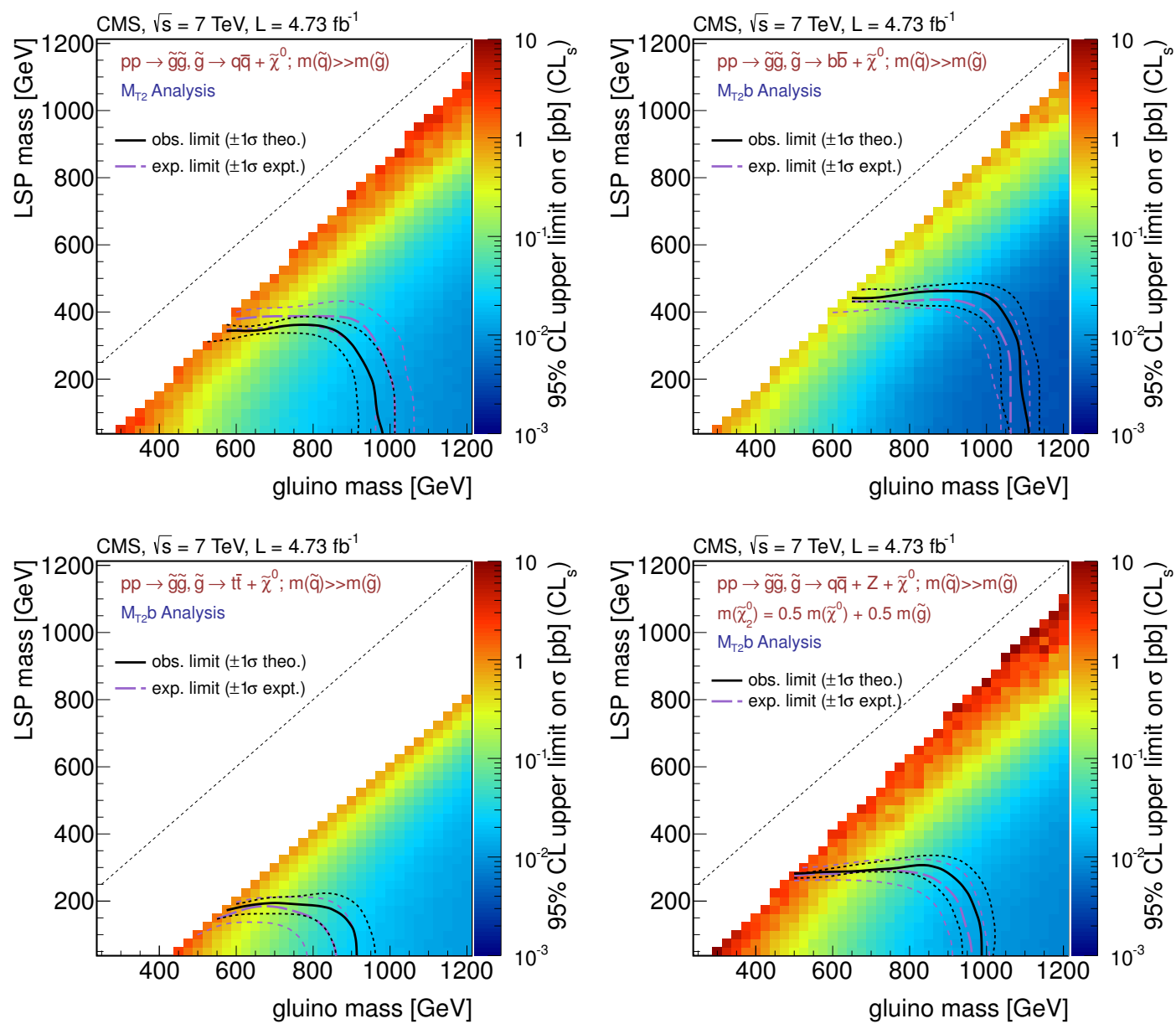

Figure 6. Exclusion limits for simplified model spectra. Upper left: gluino pair production with $\widetilde{\mathrm{g}} \rightarrow \mathrm{q} \overline{\mathrm{q}} \widetilde{\chi}^{0}$ using the $M_{\mathrm{T} 2}$ analysis. Upper right: gluino pair production with $\widetilde{\mathrm{g}} \rightarrow \mathrm{b} \overline{\mathrm{b}} \widetilde{\chi}^{0}$, using the $M_{\mathrm{T} 2} b$ analysis. Lower left: gluino pair production with $\widetilde{\mathrm{g}} \rightarrow \mathrm{t} \overline{\mathrm{t}} \widetilde{\chi}^{0}$, using the $M_{\mathrm{T} 2} b$ analysis. Lower right: gluino pair production with $\widetilde{\mathrm{g}} \rightarrow \mathrm{q} \overline{\mathrm{q}} \widetilde{\chi}^{0}$, using the $M_{\mathrm{T} 2} b$ analysis. The signal production cross sections are calculated at NLO and NLL accuracy $[26,45,46]$.

\section{Summary}

We have conducted a search for supersymmetry or similar new physics in hadronic final states using the $M_{\mathrm{T} 2}$ variable calculated from massless pseudojets. $M_{\mathrm{T} 2}$ is strongly correlated with $E_{\mathrm{T}}^{\mathrm{miss}}$ for SUSY processes, yet provides a natural suppression of QCD multijet background. The data set for this analysis corresponds to $4.73 \mathrm{fb}^{-1}$ of integrated luminosity in $\sqrt{s}=7 \mathrm{TeV}$ pp collisions collected with the CMS detector during the 2011 LHC run. All candidate events are selected using hadronic triggers. Two complementary analyses are performed. The $M_{\mathrm{T} 2}$ analysis targets decays of moderately heavy squarks and gluinos, which naturally feature a sizeable $E_{\mathrm{T}}^{\text {miss }}$. This analysis is based on events containing three or more jets and no isolated leptons. We show that the tail of the $M_{\mathrm{T} 2}$ distribution, obtained after this selection, is sensitive to a potential SUSY signal. A second approach, the $M_{\mathrm{T} 2} b$ analysis, is designed to increase the sensitivity to events with heavy squarks and light 
gluinos, in which the $E_{\mathrm{T}}^{\text {miss }}$ tends to be smaller. Therefore, the restriction on $M_{\mathrm{T} 2}$ is relaxed. The effect of the loosened $M_{\mathrm{T} 2}$ is compensated by requiring at least one b-tagged jet and a larger jet multiplicity, to suppress the QCD multijet background. For both analyses, the standard model backgrounds, arising from QCD multijet, electroweak, and top-quark production processes, are obtained from data control samples and simulation. No excess beyond the standard model expectations is found. Exclusion limits are established in the CMSSM parameter space, as well as for some simplified model spectra. Conservatively, using the minus one standard deviation $(-1 \sigma)$ theory uncertainty values, absolute mass limits in the CMSSM scenario for $\tan \beta=10$ are found to be $m(\widetilde{\mathrm{q}})>1110 \mathrm{GeV}$ and $m(\widetilde{\mathrm{g}})>800 \mathrm{GeV}$, and $m(\widetilde{\mathrm{q}})=m(\widetilde{\mathrm{g}})>1180 \mathrm{GeV}$ assuming equal squark and gluino masses.

\section{Acknowledgments}

We congratulate our colleagues in the CERN accelerator departments for the excellent performance of the LHC machine. We thank the technical and administrative staff at CERN and other CMS institutes, and acknowledge support from: FMSR (Austria); FNRS and FWO (Belgium); CNPq, CAPES, FAPERJ, and FAPESP (Brazil); MES (Bulgaria); CERN; CAS, MoST, and NSFC (China); COLCIENCIAS (Colombia); MSES (Croatia); RPF (Cyprus); MoER, SF0690030s09 and ERDF (Estonia); Academy of Finland, MEC, and HIP (Finland); CEA and CNRS/IN2P3 (France); BMBF, DFG, and HGF (Germany); GSRT (Greece); OTKA and NKTH (Hungary); DAE and DST (India); IPM (Iran); SFI (Ireland); INFN (Italy); NRF and WCU (Korea); LAS (Lithuania); CINVESTAV, CONACYT, SEP, and UASLP-FAI (Mexico); MSI (New Zealand); PAEC (Pakistan); MSHE and NSC (Poland); FCT (Portugal); JINR (Armenia, Belarus, Georgia, Ukraine, Uzbekistan); MON, RosAtom, RAS and RFBR (Russia); MSTD (Serbia); SEIDI and CPAN (Spain); Swiss Funding Agencies (Switzerland); NSC (Taipei); TUBITAK and TAEK (Turkey); STFC (United Kingdom); DOE and NSF (USA).

Individuals have received support from the Marie-Curie programme and the European Research Council (European Union); the Leventis Foundation; the A. P. Sloan Foundation; the Alexander von Humboldt Foundation; the Belgian Federal Science Policy Office; the Fonds pour la Formation à la Recherche dans l'Industrie et dans l'Agriculture (FRIABelgium); the Agentschap voor Innovatie door Wetenschap en Technologie (IWT-Belgium); the Council of Science and Industrial Research, India; the Iran National Science Foundation (INSF); the Compagnia di San Paolo (Torino); and the HOMING PLUS programme of Foundation for Polish Science, cofinanced from the European Union, Regional Development Fund.

Open Access. This article is distributed under the terms of the Creative Commons Attribution License which permits any use, distribution and reproduction in any medium, provided the original author(s) and source are credited.

\section{References}

[1] S.P. Martin, A supersymmetry primer, hep-ph/9709356 [INSPIRE]. 
[2] C. Lester and D. Summers, Measuring masses of semiinvisibly decaying particles pair produced at hadron colliders, Phys. Lett. B 463 (1999) 99 [hep-ph/9906349] [INSPIRE].

[3] A. Barr, C. Lester and P. Stephens, $M_{\mathrm{T} 2}$ : the truth behind the glamour, J. Phys. G 29 (2003) 2343 [hep-ph/0304226] [INSPIRE].

[4] CMS collaboration, S. Chatrchyan et al., Search for new physics with jets and missing transverse momentum in pp collisions at $\sqrt{s}=7$ TeV, JHEP 08 (2011) 155 [arXiv: 1106.4503] [INSPIRE].

[5] CMS collaboration, S. Chatrchyan et al., Inclusive search for squarks and gluinos in pp collisions at $\sqrt{s}=7 \mathrm{TeV}$, Phys. Rev. D 85 (2012) 012004 [arXiv:1107.1279] [INSPIRE].

[6] CMS collaboration, V. Khachatryan et al., Search for supersymmetry in pp collisions at 7 $\mathrm{TeV}$ in events with jets and missing transverse energy, Phys. Lett. B 698 (2011) 196 [arXiv: 1101.1628] [INSPIRE].

[7] CMS collaboration, S. Chatrchyan et al., Search for supersymmetry at the LHC in events with jets and missing transverse energy, Phys. Rev. Lett. 107 (2011) 221804 [arXiv: 1109.2352] [INSPIRE].

[8] ATLAS collaboration, G. Aad et al., Search for squarks and gluinos using final states with jets and missing transverse momentum with the ATLAS detector in $\sqrt{s}=7 \mathrm{TeV}$ proton-proton collisions, Phys. Lett. B 710 (2012) 67 [arXiv:1109.6572] [INSPIRE].

[9] ATLAS collaboration, G. Aad et al., Search for squarks and gluinos using final states with jets and missing transverse momentum with the ATLAS detector in $\sqrt{s}=7 \mathrm{TeV}$ proton-proton collisions, Phys. Lett. B 701 (2011) 186 [arXiv:1102.5290] [INSPIRE].

[10] ATLAS collaboration, G. Aad et al., Search for new phenomena in final states with large jet multiplicities and missing transverse momentum using $\sqrt{s}=7 \mathrm{TeV}$ pp collisions with the ATLAS detector, JHEP 11 (2011) 099 [arXiv:1110.2299] [INSPIRE].

[11] ATLAS collaboration, G. Aad et al., Hunt for new phenomena using large jet multiplicities and missing transverse momentum with ATLAS in $4.7 \mathrm{fb}^{-1}$ of $\sqrt{\mathrm{s}}=7 \mathrm{TeV}$ proton-proton collisions, JHEP 07 (2012) 167 [arXiv:1206.1760] [INSPIRE].

[12] UA1 collaboration, G. Arnison et al., Experimental observation of isolated large transverse energy electrons with associated missing energy at $\sqrt{s}=540 \mathrm{GeV}$, Phys. Lett. B 122 (1983) 103 [INSPIRE].

[13] W.S. Cho, K. Choi, Y.G. Kim and C.B. Park, Measuring superparticle masses at hadron collider using the transverse mass kink, JHEP 02 (2008) 035 [arXiv:0711.4526] [INSPIRE].

[14] M. Burns, K. Kong, K.T. Matchev and M. Park, Using subsystem $M_{\mathrm{T} 2}$ for complete mass determinations in decay chains with missing energy at hadron colliders, JHEP 03 (2009) 143 [arXiv: 0810.5576] [INSPIRE].

[15] H.-C. Cheng and Z. Han, Minimal kinematic constraints and $M_{\mathrm{T} 2}$, JHEP 12 (2008) 063 [arXiv:0810.5178] [INSPIRE].

[16] A.J. Barr and C. Gwenlan, The race for supersymmetry: using $M_{\mathrm{T} 2}$ for discovery, Phys. Rev. D 80 (2009) 074007 [arXiv:0907.2713] [INSPIRE].

[17] CMS collaboration, G. Bayatian et al., CMS technical design report, volume II: physics performance, J. Phys. G 34 (2007) 995 [INSPIRE]. 
[18] T. Sjöstrand, S. Mrenna and P.Z. Skands, PYTHIA 6.4 physics and manual, JHEP 05 (2006) 026 [hep-ph/0603175] [INSPIRE].

[19] CMS collaboration, The CMS experiment at the CERN LHC, 2008 JINST 3 S08004.

[20] J. Alwall, M. Herquet, F. Maltoni, O. Mattelaer and T. Stelzer, MadGraph 5: going beyond, JHEP 06 (2011) 128 [arXiv:1106.0522] [INSPIRE].

[21] GEANT4 collaboration, GEANT4: a simulation toolkit, Nucl. Instrum. Meth. A 506 (2003) 250.

[22] B. Allanach, SOFTSUSY: a program for calculating supersymmetric spectra, Comput. Phys. Commun. 143 (2002) 305 [hep-ph/0104145] [INSPIRE].

[23] M. Muhlleitner, A. Djouadi and Y. Mambrini, SDECAY: a Fortran code for the decays of the supersymmetric particles in the MSSM, Comput. Phys. Commun. 168 (2005) 46 [hep-ph/0311167] [INSPIRE].

[24] G.L. Kane, C.F. Kolda, L. Roszkowski and J.D. Wells, Study of constrained minimal supersymmetry, Phys. Rev. D 49 (1994) 6173 [hep-ph/9312272] [INSPIRE].

[25] J. Pumplin et al., New generation of parton distributions with uncertainties from global QCD analysis, JHEP 07 (2002) 012 [hep-ph/0201195] [INSPIRE].

[26] W. Beenakker, R. Hopker and M. Spira, PROSPINO: a program for the production of supersymmetric particles in next-to-leading order QCD, hep-ph/9611232 [INSPIRE].

[27] CMS collaboration, Particle-flow event reconstruction in CMS and performance for jets, taus and MET, PAS-PFT-09-001 (2009).

[28] CMS collaboration, Electron reconstruction and identification at $\sqrt{s}=7 \mathrm{TeV}$, PAS-EGM-10-004 (2010).

[29] CMS collaboration, Particle-flow commissioning with muons and electrons from $J / \Psi$ and $W$ events at $7 \mathrm{TeV}$, PAS-PFT-10-003 (2010).

[30] CMS collaboration, Performance of muon identification in pp collisions at $\sqrt{s}=7 \mathrm{TeV}$, PAS-MUO-10-002 (2010).

[31] M. Cacciari, G.P. Salam and G. Soyez, The anti- $k_{t}$ jet clustering algorithm, JHEP 04 (2008) 063 [arXiv:0802.1189] [INSPIRE].

[32] CMS collaboration, Jet performance in pp collisions at 7 TeV, PAS-JME-10-003 (2010).

[33] CMS collaboration, Commissioning of the particle-flow reconstruction in minimum-bias and jet events from pp collisions at $7 \mathrm{TeV}$, PAS-PFT-10-002 (2010).

[34] CMS collaboration, S. Chatrchyan et al., Determination of jet energy calibration and transverse momentum resolution in CMS, 2011 JINST 6 P11002 [arXiv:1107.4277] [INSPIRE].

[35] M. Cacciari and G.P. Salam, Pileup subtraction using jet areas, Phys. Lett. B 659 (2008) 119 [arXiv:0707.1378] [INSPIRE].

[36] M. Cacciari, G.P. Salam and G. Soyez, The catchment area of jets, JHEP 04 (2008) 005 [arXiv: 0802.1188] [INSPIRE].

[37] CMS collaboration, Commissioning of b-jet identification with pp collisions at $\sqrt{s}=7 \mathrm{TeV}$, PAS-BTV-10-001 (2010). 
[38] CMS collaboration, Tracking and primary vertex results in first $7 \mathrm{TeV}$ collisions, PAS-TRK-10-005 (2010).

[39] CMS collaboration, Tau identification in CMS, CMS-PAS-PFT-11-001 (2011).

[40] CMS collaboration, Isolated photon reconstruction and identification at $\sqrt{s}=7 \mathrm{TeV}$, PAS-EGM-10-006 (2010).

[41] ATLAS and CMS collaborations, Procedure for the LHC Higgs boson search combination in summer 2011, ATL-PHYS-PUB-2011-11 (2011).

[42] T. Junk, Confidence level computation for combining searches with small statistics, Nucl. Instrum. Meth. A 434 (1999) 435 [hep-ex/9902006] [INSPIRE].

[43] A.L. Read, Presentation of search results: the $C L_{\mathrm{s}}$ technique, J. Phys. G 28 (2002) 2693 [INSPIRE].

[44] K. Matchev and R. Remington, Updated templates for the interpretation of LHC results on supersymmetry in the context of mSUGRA, arXiv:1202.6580 [INSPIRE].

[45] A. Kulesza and L. Motyka, Threshold resummation for squark-antisquark and gluino-pair production at the LHC, Phys. Rev. Lett. 102 (2009) 111802 [arXiv:0807.2405] [INSPIRE].

[46] M. Krämer et al., Supersymmetry production cross sections in pp collisions at $\sqrt{s}=7 \mathrm{TeV}$, arXiv:1206.2892 [INSPIRE].

[47] CMS collaboration, Absolute calibration of the luminosity measurement at CMS: winter 2012 update, PAS-SMP-12-008 (2012).

[48] M. Botje et al., The PDF4LHC working group interim recommendations, arXiv:1101.0538 [INSPIRE].

[49] LHC New Physics Working Group collaboration, D. Alves et al., Simplified models for LHC new physics searches, J. Phys. G 39 (2012) 105005 [arXiv:1105.2838] [INSPIRE]. 


\section{The CMS collaboration}

\section{Yerevan Physics Institute, Yerevan, Armenia}

S. Chatrchyan, V. Khachatryan, A.M. Sirunyan, A. Tumasyan

\section{Institut für Hochenergiephysik der OeAW, Wien, Austria}

W. Adam, T. Bergauer, M. Dragicevic, J. Erö, C. Fabjan ${ }^{1}$, M. Friedl, R. Frühwirth ${ }^{1}$, V.M. Ghete, J. Hammer, N. Hörmann, J. Hrubec, M. Jeitler ${ }^{1}$, W. Kiesenhofer, V. Knünz, M. Krammer ${ }^{1}$, D. Liko, I. Mikulec, M. Pernicka ${ }^{\dagger}$, B. Rahbaran, C. Rohringer, H. Rohringer, R. Schöfbeck, J. Strauss, A. Taurok, P. Wagner, W. Waltenberger, G. Walzel, E. Widl, C.-E. Wulz ${ }^{1}$

\section{National Centre for Particle and High Energy Physics, Minsk, Belarus}

V. Mossolov, N. Shumeiko, J. Suarez Gonzalez

\section{Universiteit Antwerpen, Antwerpen, Belgium}

S. Bansal, T. Cornelis, E.A. De Wolf, X. Janssen, S. Luyckx, L. Mucibello, S. Ochesanu, B. Roland, R. Rougny, M. Selvaggi, Z. Staykova, H. Van Haevermaet, P. Van Mechelen, N. Van Remortel, A. Van Spilbeeck

\section{Vrije Universiteit Brussel, Brussel, Belgium}

F. Blekman, S. Blyweert, J. D'Hondt, R. Gonzalez Suarez, A. Kalogeropoulos, M. Maes, A. Olbrechts, W. Van Doninck, P. Van Mulders, G.P. Van Onsem, I. Villella

\section{Université Libre de Bruxelles, Bruxelles, Belgium}

B. Clerbaux, G. De Lentdecker, V. Dero, A.P.R. Gay, T. Hreus, A. Léonard, P.E. Marage, T. Reis, L. Thomas, C. Vander Velde, P. Vanlaer, J. Wang

\section{Ghent University, Ghent, Belgium}

V. Adler, K. Beernaert, A. Cimmino, S. Costantini, G. Garcia, M. Grunewald, B. Klein, J. Lellouch, A. Marinov, J. Mccartin, A.A. Ocampo Rios, D. Ryckbosch, N. Strobbe, F. Thyssen, M. Tytgat, P. Verwilligen, S. Walsh, E. Yazgan, N. Zaganidis

\section{Université Catholique de Louvain, Louvain-la-Neuve, Belgium}

S. Basegmez, G. Bruno, R. Castello, A. Caudron, L. Ceard, C. Delaere, T. du Pree, D. Favart, L. Forthomme, A. Giammanco ${ }^{2}$, J. Hollar, V. Lemaitre, J. Liao, O. Militaru, C. Nuttens, D. Pagano, L. Perrini, A. Pin, K. Piotrzkowski, N. Schul, J.M. Vizan Garcia

Université de Mons, Mons, Belgium

N. Beliy, T. Caebergs, E. Daubie, G.H. Hammad

\section{Centro Brasileiro de Pesquisas Fisicas, Rio de Janeiro, Brazil}

G.A. Alves, M. Correa Martins Junior, D. De Jesus Damiao, T. Martins, M.E. Pol, M.H.G. Souza

\section{Universidade do Estado do Rio de Janeiro, Rio de Janeiro, Brazil}

W.L. Aldá Júnior, W. Carvalho, A. Custódio, E.M. Da Costa, C. De Oliveira Martins, S. Fonseca De Souza, D. Matos Figueiredo, L. Mundim, H. Nogima, V. Oguri, W.L. Prado Da Silva, A. Santoro, L. Soares Jorge, A. Sznajder 
Instituto de Fisica Teorica, Universidade Estadual Paulista, Sao Paulo, Brazil C.A. Bernardes ${ }^{3}$, F.A. Dias ${ }^{4}$, T.R. Fernandez Perez Tomei, E. M. Gregores ${ }^{3}$, C. Lagana, F. Marinho, P.G. Mercadante ${ }^{3}$, S.F. Novaes, Sandra S. Padula

Institute for Nuclear Research and Nuclear Energy, Sofia, Bulgaria

V. Genchev ${ }^{5}$, P. Iaydjiev ${ }^{5}$, S. Piperov, M. Rodozov, S. Stoykova, G. Sultanov, V. Tcholakov,

R. Trayanov, M. Vutova

University of Sofia, Sofia, Bulgaria

A. Dimitrov, R. Hadjiiska, V. Kozhuharov, L. Litov, B. Pavlov, P. Petkov

Institute of High Energy Physics, Beijing, China

J.G. Bian, G.M. Chen, H.S. Chen, C.H. Jiang, D. Liang, S. Liang, X. Meng, J. Tao, J. Wang, X. Wang, Z. Wang, H. Xiao, M. Xu, J. Zang, Z. Zhang

State Key Lab. of Nucl. Phys. and Tech., Peking University, Beijing, China C. Asawatangtrakuldee, Y. Ban, S. Guo, Y. Guo, W. Li, S. Liu, Y. Mao, S.J. Qian, H. Teng, S. Wang, B. Zhu, W. Zou

Universidad de Los Andes, Bogota, Colombia

C. Avila, J.P. Gomez, B. Gomez Moreno, A.F. Osorio Oliveros, J.C. Sanabria

Technical University of Split, Split, Croatia

N. Godinovic, D. Lelas, R. Plestina ${ }^{6}$, D. Polic, I. Puljak ${ }^{5}$

University of Split, Split, Croatia

Z. Antunovic, M. Kovac

Institute Rudjer Boskovic, Zagreb, Croatia

V. Brigljevic, S. Duric, K. Kadija, J. Luetic, S. Morovic

University of Cyprus, Nicosia, Cyprus

A. Attikis, M. Galanti, G. Mavromanolakis, J. Mousa, C. Nicolaou, F. Ptochos, P.A. Razis

Charles University, Prague, Czech Republic

M. Finger, M. Finger Jr.

Academy of Scientific Research and Technology of the Arab Republic of Egypt, Egyptian Network of High Energy Physics, Cairo, Egypt

Y. Assran ${ }^{7}$, S. Elgammal ${ }^{8}$, A. Ellithi Kamel ${ }^{9}$, S. Khalil ${ }^{8}$, M.A. Mahmoud ${ }^{10}$, A. Radi ${ }^{11,12}$

National Institute of Chemical Physics and Biophysics, Tallinn, Estonia

M. Kadastik, M. Müntel, M. Raidal, L. Rebane, A. Tiko

Department of Physics, University of Helsinki, Helsinki, Finland

V. Azzolini, P. Eerola, G. Fedi, M. Voutilainen

Helsinki Institute of Physics, Helsinki, Finland

J. Härkönen, A. Heikkinen, V. Karimäki, R. Kinnunen, M.J. Kortelainen, T. Lampén,

K. Lassila-Perini, S. Lehti, T. Lindén, P. Luukka, T. Mäenpää, T. Peltola, E. Tuominen,

J. Tuominiemi, E. Tuovinen, D. Ungaro, L. Wendland 
Lappeenranta University of Technology, Lappeenranta, Finland

K. Banzuzi, A. Karjalainen, A. Korpela, T. Tuuva

DSM/IRFU, CEA/Saclay, Gif-sur-Yvette, France

M. Besancon, S. Choudhury, M. Dejardin, D. Denegri, B. Fabbro, J.L. Faure, F. Ferri, S. Ganjour, A. Givernaud, P. Gras, G. Hamel de Monchenault, P. Jarry, E. Locci, J. Malcles, L. Millischer, A. Nayak, J. Rander, A. Rosowsky, I. Shreyber, M. Titov

Laboratoire Leprince-Ringuet, Ecole Polytechnique, IN2P3-CNRS, Palaiseau, France

S. Baffioni, F. Beaudette, L. Benhabib, L. Bianchini, M. Bluj ${ }^{13}$, C. Broutin, P. Busson, C. Charlot, N. Daci, T. Dahms, L. Dobrzynski, R. Granier de Cassagnac, M. Haguenauer, P. Miné, C. Mironov, M. Nguyen, C. Ochando, P. Paganini, D. Sabes, R. Salerno, Y. Sirois, C. Veelken, A. Zabi

Institut Pluridisciplinaire Hubert Curien, Université de Strasbourg, Université de Haute Alsace Mulhouse, CNRS/IN2P3, Strasbourg, France

J.-L. Agram ${ }^{14}$, J. Andrea, D. Bloch, D. Bodin, J.-M. Brom, M. Cardaci, E.C. Chabert, C. Collard, E. Conte ${ }^{14}$, F. Drouhin ${ }^{14}$, C. Ferro, J.-C. Fontaine ${ }^{14}$, D. Gelé, U. Goerlach, P. Juillot, A.-C. Le Bihan, P. Van Hove

Centre de Calcul de l'Institut National de Physique Nucleaire et de Physique des Particules (IN2P3), Villeurbanne, France

F. Fassi, D. Mercier

Université de Lyon, Université Claude Bernard Lyon 1, CNRS-IN2P3, Institut de Physique Nucléaire de Lyon, Villeurbanne, France

S. Beauceron, N. Beaupere, O. Bondu, G. Boudoul, J. Chasserat, R. Chierici ${ }^{5}$, D. Contardo, P. Depasse, H. El Mamouni, J. Fay, S. Gascon, M. Gouzevitch, B. Ille, T. Kurca, M. Lethuillier, L. Mirabito, S. Perries, V. Sordini, S. Tosi, Y. Tschudi, P. Verdier, S. Viret

E. Andronikashvili Institute of Physics, Academy of Science, Tbilisi, Georgia L. Rurua

RWTH Aachen University, I. Physikalisches Institut, Aachen, Germany

G. Anagnostou, S. Beranek, M. Edelhoff, L. Feld, N. Heracleous, O. Hindrichs, R. Jussen,

K. Klein, J. Merz, A. Ostapchuk, A. Perieanu, F. Raupach, J. Sammet, S. Schael, D. Sprenger, H. Weber, B. Wittmer, V. Zhukov ${ }^{15}$

RWTH Aachen University, III. Physikalisches Institut A, Aachen, Germany M. Ata, J. Caudron, E. Dietz-Laursonn, D. Duchardt, M. Erdmann, R. Fischer, A. Güth, T. Hebbeker, C. Heidemann, K. Hoepfner, D. Klingebiel, P. Kreuzer, J. Lingemann, C. Magass, M. Merschmeyer, A. Meyer, M. Olschewski, P. Papacz, H. Pieta, H. Reithler, S.A. Schmitz, L. Sonnenschein, J. Steggemann, D. Teyssier, M. Weber

RWTH Aachen University, III. Physikalisches Institut B, Aachen, Germany

M. Bontenackels, V. Cherepanov, G. Flügge, H. Geenen, M. Geisler, W. Haj Ahmad, F. Hoehle, B. Kargoll, T. Kress, Y. Kuessel, A. Nowack, L. Perchalla, O. Pooth, J. Rennefeld, P. Sauerland, A. Stahl 


\section{Deutsches Elektronen-Synchrotron, Hamburg, Germany}

M. Aldaya Martin, J. Behr, W. Behrenhoff, U. Behrens, M. Bergholz ${ }^{16}$, A. Bethani, K. Borras, A. Burgmeier, A. Cakir, L. Calligaris, A. Campbell, E. Castro, F. Costanza, D. Dammann, C. Diez Pardos, G. Eckerlin, D. Eckstein, G. Flucke, A. Geiser, I. Glushkov, P. Gunnellini, S. Habib, J. Hauk, G. Hellwig, H. Jung, M. Kasemann, P. Katsas, C. Kleinwort, H. Kluge, A. Knutsson, M. Krämer, D. Krücker, E. Kuznetsova, W. Lange, W. Lohmann ${ }^{16}$, B. Lutz, R. Mankel, I. Marfin, M. Marienfeld, I.-A. MelzerPellmann, A.B. Meyer, J. Mnich, A. Mussgiller, S. Naumann-Emme, J. Olzem, H. Perrey, A. Petrukhin, D. Pitzl, A. Raspereza, P.M. Ribeiro Cipriano, C. Riedl, E. Ron, M. Rosin, J. Salfeld-Nebgen, R. Schmidt ${ }^{16}$, T. Schoerner-Sadenius, N. Sen, A. Spiridonov, M. Stein, R. Walsh, C. Wissing

\section{University of Hamburg, Hamburg, Germany}

C. Autermann, V. Blobel, J. Draeger, H. Enderle, J. Erfle, U. Gebbert, M. Görner, T. Hermanns, R.S. Höing, K. Kaschube, G. Kaussen, H. Kirschenmann, R. Klanner, J. Lange, B. Mura, F. Nowak, T. Peiffer, N. Pietsch, D. Rathjens, C. Sander, H. Schettler, P. Schleper, E. Schlieckau, A. Schmidt, M. Schröder, T. Schum, M. Seidel, V. Sola, H. Stadie, G. Steinbrück, J. Thomsen, L. Vanelderen

Institut für Experimentelle Kernphysik, Karlsruhe, Germany

C. Barth, J. Berger, C. Böser, T. Chwalek, W. De Boer, A. Descroix, A. Dierlamm, M. Feindt, M. Guthoff ${ }^{5}$, C. Hackstein, F. Hartmann, T. Hauth ${ }^{5}$, M. Heinrich, H. Held, K.H. Hoffmann, S. Honc, I. Katkov ${ }^{15}$, J.R. Komaragiri, P. Lobelle Pardo, D. Martschei, S. Mueller, Th. Müller, M. Niegel, A. Nürnberg, O. Oberst, A. Oehler, J. Ott, G. Quast, K. Rabbertz, F. Ratnikov, N. Ratnikova, S. Röcker, A. Scheurer, F.-P. Schilling, G. Schott, H.J. Simonis, F.M. Stober, D. Troendle, R. Ulrich, J. Wagner-Kuhr, S. Wayand, T. Weiler, M. Zeise

Institute of Nuclear Physics "Demokritos", Aghia Paraskevi, Greece

G. Daskalakis, T. Geralis, S. Kesisoglou, A. Kyriakis, D. Loukas, I. Manolakos, A. Markou, C. Markou, C. Mavrommatis, E. Ntomari

University of Athens, Athens, Greece

L. Gouskos, T.J. Mertzimekis, A. Panagiotou, N. Saoulidou

University of Ioánnina, Ioánnina, Greece

I. Evangelou, C. Foudas ${ }^{5}$, P. Kokkas, N. Manthos, I. Papadopoulos, V. Patras

KFKI Research Institute for Particle and Nuclear Physics, Budapest, Hungary G. Bencze, C. Hajdu ${ }^{5}$, P. Hidas, D. Horvath ${ }^{17}$, F. Sikler, V. Veszpremi, G. Vesztergombi ${ }^{18}$

Institute of Nuclear Research ATOMKI, Debrecen, Hungary

N. Beni, S. Czellar, J. Molnar, J. Palinkas, Z. Szillasi

University of Debrecen, Debrecen, Hungary

J. Karancsi, P. Raics, Z.L. Trocsanyi, B. Ujvari 
Panjab University, Chandigarh, India

S.B. Beri, V. Bhatnagar, N. Dhingra, R. Gupta, M. Jindal, M. Kaur, M.Z. Mehta, N. Nishu, L.K. Saini, A. Sharma, J. Singh

University of Delhi, Delhi, India

Ashok Kumar, Arun Kumar, S. Ahuja, A. Bhardwaj, B.C. Choudhary, S. Malhotra, M. Naimuddin, K. Ranjan, V. Sharma, R.K. Shivpuri

Saha Institute of Nuclear Physics, Kolkata, India

S. Banerjee, S. Bhattacharya, S. Dutta, B. Gomber, Sa. Jain, Sh. Jain, R. Khurana, S. Sarkar, M. Sharan

Bhabha Atomic Research Centre, Mumbai, India

A. Abdulsalam, R.K. Choudhury, D. Dutta, S. Kailas, V. Kumar, P. Mehta, A.K. Mohanty ${ }^{5}$, L.M. Pant, P. Shukla

Tata Institute of Fundamental Research - EHEP, Mumbai, India

T. Aziz, S. Ganguly, M. Guchait ${ }^{19}$, M. Maity ${ }^{20}$, G. Majumder, K. Mazumdar, G.B. Mohanty, B. Parida, K. Sudhakar, N. Wickramage

Tata Institute of Fundamental Research - HECR, Mumbai, India

S. Banerjee, S. Dugad

Institute for Research in Fundamental Sciences (IPM), Tehran, Iran

H. Arfaei, H. Bakhshiansohi ${ }^{21}$, S.M. Etesami ${ }^{22}$, A. Fahim ${ }^{21}$, M. Hashemi, H. Hesari,

A. Jafari ${ }^{21}$, M. Khakzad, M. Mohammadi Najafabadi, S. Paktinat Mehdiabadi, B. Safarzadeh ${ }^{23}$, M. Zeinali ${ }^{22}$

INFN Sezione di Bari ${ }^{a}$, Università di Bari ${ }^{b}$, Politecnico di Bari ${ }^{c}$, Bari, Italy M. Abbrescia $^{a, b}$, L. Barbone ${ }^{a, b}$, C. Calabria ${ }^{a, b, 5}$, S.S. Chhibra ${ }^{a, b}$, A. Colaleo ${ }^{a}$, D. Creanza ${ }^{a, c}$, N. De Filippis ${ }^{a, c, 5}$, M. De Palma ${ }^{a, b}$, L. Fiore ${ }^{a}$, G. Iasellia ${ }^{a, c}$, L. Lusito ${ }^{a, b}$, G. Maggi ${ }^{a, c}$, M. Maggi ${ }^{a}$, B. Marangelli ${ }^{a, b}$, S. My ${ }^{a, c}$, S. Nuzzo ${ }^{a, b}$, N. Pacifico ${ }^{a, b}$,

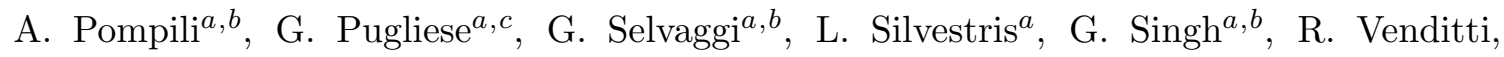
G. Zito ${ }^{a}$

INFN Sezione di Bologna ${ }^{a}$, Università di Bologna ${ }^{b}$, Bologna, Italy

G. Abbiendi $^{a}$, A.C. Benvenuti ${ }^{a}$, D. Bonacorsi ${ }^{a}, b$ S. Braibant-Giacomelli ${ }^{a, b}$, L. Brigliadori ${ }^{a, b}$, P. Capiluppi ${ }^{a, b}$, A. Castro ${ }^{a, b}$, F.R. Cavallo ${ }^{a}$, M. Cuffiani ${ }^{a, b}$, G.M. Dallavalle ${ }^{a}$, F. Fabbri ${ }^{a}$, A. Fanfani ${ }^{a, b}$, D. Fasanella ${ }^{a, b, 5}$, P. Giacomelli ${ }^{a}$, C. Grandi ${ }^{a}$, L. Guiducci ${ }^{a}, b$, S. Marcellini ${ }^{a}$, G. Masetti ${ }^{a}$, M. Meneghelli ${ }^{a, b, 5}$, A. Montanari $^{a}$,

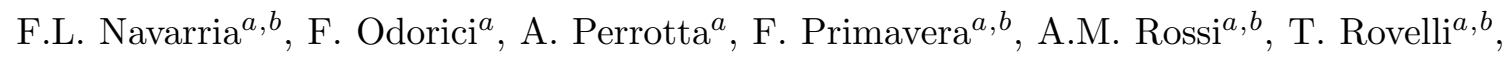
G. Siroli ${ }^{a, b}$, R. Travaglini ${ }^{a, b}$

INFN Sezione di Catania ${ }^{a}$, Università di Catania ${ }^{b}$, Catania, Italy

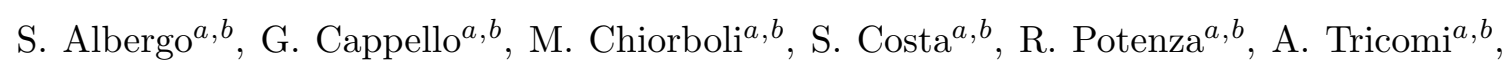
C. Tuve ${ }^{a, b}$ 
INFN Sezione di Firenze ${ }^{a}$, Università di Firenze ${ }^{b}$, Firenze, Italy

G. Barbagli ${ }^{a}$, V. Ciulli ${ }^{a}, b$, C. Civinini ${ }^{a}$, R. D’Alessandro ${ }^{a, b}$, E. Focardi ${ }^{a}, b$, S. Frosali ${ }^{a, b}$,

E. Gallo ${ }^{a}$, S. Gonzi ${ }^{a}, b$, M. Meschini ${ }^{a}$, S. Paoletti ${ }^{a}$, G. Sguazzoni ${ }^{a}$, A. Tropiano ${ }^{a, 5}$

INFN Laboratori Nazionali di Frascati, Frascati, Italy

L. Benussi, S. Bianco, S. Colafranceschi ${ }^{24}$, F. Fabbri, D. Piccolo

INFN Sezione di Genova, Genova, Italy

P. Fabbricatore, R. Musenich

INFN Sezione di Milano-Bicocca ${ }^{a}$, Università di Milano-Bicocca ${ }^{b}$, Milano, Italy

A. Benaglia ${ }^{a, b, 5}$, F. De Guio ${ }^{a, b}$, L. Di Matteo ${ }^{a, b, 5}$, S. Fiorendi $^{a, b}$, S. Gennai $^{a, 5}$, A. Ghezzi $^{a, b}$, S. Malvezzi ${ }^{a}$, R.A. Manzoni ${ }^{a, b}$, A. Martelli ${ }^{a}, b$, A. Massironi ${ }^{a, b, 5}$, D. Menasce $^{a}$, L. Moroni ${ }^{a}$, M. Paganoni ${ }^{a, b}$, D. Pedrini ${ }^{a}$, S. Ragazzi ${ }^{a, b}$, N. Redaelli ${ }^{a}$, S. Sala ${ }^{a}$, T. Tabarelli de Fatis ${ }^{a, b}$

INFN Sezione di Napoli ${ }^{a}$, Università di Napoli "Federico II" ${ }^{b}$, Napoli, Italy S. Buontempo ${ }^{a}$, C.A. Carrillo Montoya ${ }^{a, 5}$, N. Cavallo ${ }^{a, 25}$, A. De Cosa $^{a, b, 5}$, O. Dogangun $^{a, b}$,

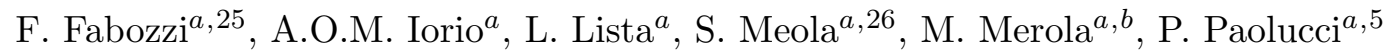

INFN Sezione di Padova ${ }^{a}$, Università di Padova ${ }^{b}$, Università di Trento (Trento) ${ }^{c}$, Padova, Italy

P. Azzi ${ }^{a}$, N. Bacchetta ${ }^{a, 5}$, P. Bellan ${ }^{a, b}$, D. Bisello ${ }^{a, b}$, A. Branca ${ }^{a, 5}$, R. Carlin $^{a, b}$, P. Checchia ${ }^{a}$, T. Dorigo ${ }^{a}$, U. Dosselli ${ }^{a}$, F. Gasparini ${ }^{a, b}$, U. Gasparini $^{a}, b$, A. Gozzelino $^{a}$, K. Kanishchev ${ }^{a, c}$, S. Lacaprara ${ }^{a}$, I. Lazzizzera ${ }^{a, c}$, M. Margoni $^{a, b}$, A.T. Meneguzzo ${ }^{a, b}$,

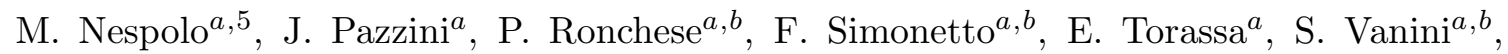
P. Zotto ${ }^{a, b}$, A. Zucchetta ${ }^{a}$, G. Zumerle ${ }^{a, b}$

INFN Sezione di Pavia ${ }^{a}$, Università di Pavia ${ }^{b}$, Pavia, Italy

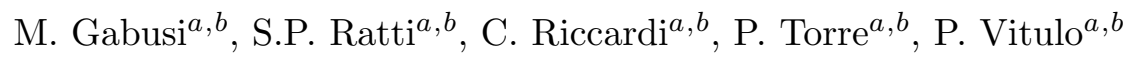

INFN Sezione di Perugia ${ }^{a}$, Università di Perugia ${ }^{b}$, Perugia, Italy

M. Biasini ${ }^{a, b}$, G.M. Bilei ${ }^{a}$, L. Fanò ${ }^{a, b}$, P. Lariccia ${ }^{a, b}$, A. Lucaroni ${ }^{a, b, 5}$, G. Mantovani ${ }^{a, b}$, M. Menichelli ${ }^{a}$, A. Nappi ${ }^{a, b}$, F. Romeo ${ }^{a, b}$, A. Saha ${ }^{a}$, A. Santocchia ${ }^{a, b}$, S. Taroni $^{a, b, 5}$

INFN Sezione di Pisa ${ }^{a}$, Università di Pisa ${ }^{b}$, Scuola Normale Superiore di Pisa ${ }^{c}$, Pisa, Italy

P. Azzurri ${ }^{a, c}$, G. Bagliesi ${ }^{a}$, T. Boccali ${ }^{a}$, G. Broccolo ${ }^{a, c}$, R. Castaldi $^{a}$, R.T. D'Agnolo $^{a, c}$,

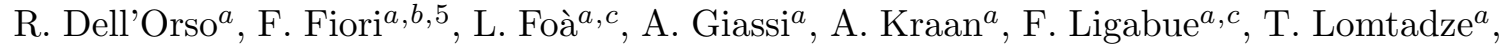
L. Martini ${ }^{a, 27}$, A. Messineo ${ }^{a, b}$, F. Palla ${ }^{a}$, A. Rizzi ${ }^{a, b}$, A.T. Serban ${ }^{a, 28}$, P. Spagnolo ${ }^{a}$,

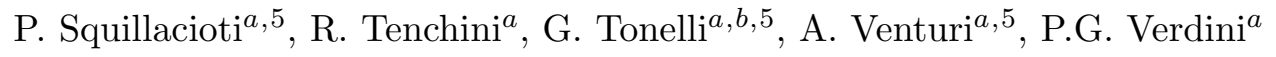

INFN Sezione di Roma ${ }^{a}$, Università di Roma "La Sapienza" ${ }^{b}$, Roma, Italy

L. Barone ${ }^{a, b}$, F. Cavallari ${ }^{a}$, D. Del Re ${ }^{a, b, 5}$, M. Diemoz ${ }^{a}$, M. Grassi ${ }^{a, b, 5}$, E. Longo ${ }^{a, b}$, P. Meridiani ${ }^{a, 5}$, F. Micheli ${ }^{a, b}$, S. Nourbakhsh ${ }^{a, b}$, G. Organtini ${ }^{a, b}$, R. Paramatti ${ }^{a}$, S. Rahatlou ${ }^{a, b}$, M. Sigamani ${ }^{a}$, L. Soffi ${ }^{a, b}$ 
INFN Sezione di Torino ${ }^{a}$, Università di Torino ${ }^{b}$, Università del Piemonte Orientale (Novara) ${ }^{c}$, Torino, Italy

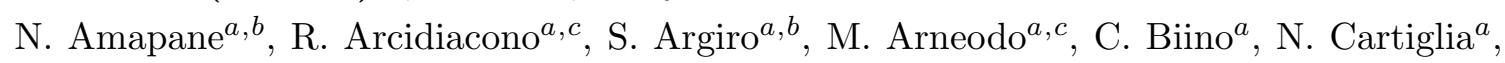
M. Costa $^{a, b}$, N. Demaria ${ }^{a}$, A. Graziano ${ }^{a, b}$, C. Mariotti ${ }^{a, 5}$, S. Maselli ${ }^{a}$, E. Migliore ${ }^{a, b}$, V. Monaco ${ }^{a, b}$, M. Musich ${ }^{a, 5}$, M.M. Obertino ${ }^{a, c}$, N. Pastrone ${ }^{a}$, M. Pelliccioni ${ }^{a}$,

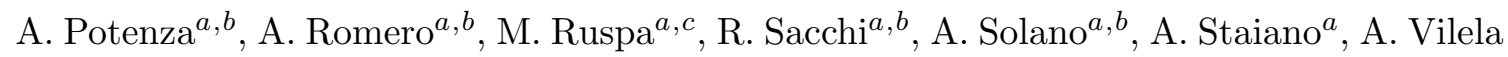
Pereira $^{a}$

INFN Sezione di Trieste ${ }^{a}$, Università di Trieste ${ }^{b}$, Trieste, Italy

S. Belforte ${ }^{a}$, V. Candelise ${ }^{a, b}$, F. Cossutti ${ }^{a}$, G. Della Ricca ${ }^{a, b}$, B. Gobbo $^{a}$, M. Marone ${ }^{a, b, 5}$, D. Montanino ${ }^{a, b, 5}$, A. Penzo $^{a}$, A. Schizzi ${ }^{a, b}$

Kangwon National University, Chunchon, Korea

S.G. Heo, T.Y. Kim, S.K. Nam

Kyungpook National University, Daegu, Korea

S. Chang, D.H. Kim, G.N. Kim, D.J. Kong, H. Park, S.R. Ro, D.C. Son, T. Son

Chonnam National University, Institute for Universe and Elementary Particles, Kwangju, Korea

J.Y. Kim, Zero J. Kim, S. Song

Korea University, Seoul, Korea

S. Choi, D. Gyun, B. Hong, M. Jo, H. Kim, T.J. Kim, K.S. Lee, D.H. Moon, S.K. Park

University of Seoul, Seoul, Korea

M. Choi, J.H. Kim, C. Park, I.C. Park, S. Park, G. Ryu

Sungkyunkwan University, Suwon, Korea

Y. Cho, Y. Choi, Y.K. Choi, J. Goh, M.S. Kim, E. Kwon, B. Lee, J. Lee, S. Lee, H. Seo, I. $\mathrm{Yu}$

Vilnius University, Vilnius, Lithuania

M.J. Bilinskas, I. Grigelionis, M. Janulis, A. Juodagalvis

Centro de Investigacion y de Estudios Avanzados del IPN, Mexico City, Mexico

H. Castilla-Valdez, E. De La Cruz-Burelo, I. Heredia-de La Cruz, R. Lopez-Fernandez,

R. Magaña Villalba, J. Martínez-Ortega, A. Sánchez-Hernández, L.M. Villasenor-Cendejas

Universidad Iberoamericana, Mexico City, Mexico

S. Carrillo Moreno, F. Vazquez Valencia

Benemerita Universidad Autonoma de Puebla, Puebla, Mexico

H.A. Salazar Ibarguen

Universidad Autónoma de San Luis Potosí, San Luis Potosí, Mexico

E. Casimiro Linares, A. Morelos Pineda, M.A. Reyes-Santos

University of Auckland, Auckland, New Zealand

D. Krofcheck 
University of Canterbury, Christchurch, New Zealand

A.J. Bell, P.H. Butler, R. Doesburg, S. Reucroft, H. Silverwood

National Centre for Physics, Quaid-I-Azam University, Islamabad, Pakistan

M. Ahmad, M.I. Asghar, H.R. Hoorani, S. Khalid, W.A. Khan, T. Khurshid, S. Qazi, M.A. Shah, M. Shoaib

Institute of Experimental Physics, Faculty of Physics, University of Warsaw, Warsaw, Poland

G. Brona, K. Bunkowski, M. Cwiok, W. Dominik, K. Doroba, A. Kalinowski, M. Konecki, J. Krolikowski

Soltan Institute for Nuclear Studies, Warsaw, Poland

H. Bialkowska, B. Boimska, T. Frueboes, R. Gokieli, M. Górski, M. Kazana, K. Nawrocki,

K. Romanowska-Rybinska, M. Szleper, G. Wrochna, P. Zalewski

Laboratório de Instrumentação e Física Experimental de Partículas, Lisboa, Portugal

N. Almeida, P. Bargassa, A. David, P. Faccioli, M. Fernandes, P.G. Ferreira Parracho,

M. Gallinaro, J. Seixas, J. Varela, P. Vischia

Joint Institute for Nuclear Research, Dubna, Russia

I. Belotelov, P. Bunin, I. Golutvin, I. Gorbunov, V. Karjavin, V. Konoplyanikov, G. Kozlov,

A. Lanev, A. Malakhov, P. Moisenz, V. Palichik, V. Perelygin, M. Savina, S. Shmatov,

V. Smirnov, A. Volodko, A. Zarubin

Petersburg Nuclear Physics Institute, Gatchina (St Petersburg), Russia

S. Evstyukhin, V. Golovtsov, Y. Ivanov, V. Kim, P. Levchenko, V. Murzin, V. Oreshkin,

I. Smirnov, V. Sulimov, L. Uvarov, S. Vavilov, A. Vorobyev, An. Vorobyev

Institute for Nuclear Research, Moscow, Russia

Yu. Andreev, A. Dermenev, S. Gninenko, N. Golubev, M. Kirsanov, N. Krasnikov, V. Matveev, A. Pashenkov, D. Tlisov, A. Toropin

Institute for Theoretical and Experimental Physics, Moscow, Russia

V. Epshteyn, M. Erofeeva, V. Gavrilov, M. Kossov ${ }^{5}$, N. Lychkovskaya, V. Popov, G. Safronov, S. Semenov, V. Stolin, E. Vlasov, A. Zhokin

Moscow State University, Moscow, Russia

A. Belyaev, E. Boos, M. Dubinin ${ }^{4}$, L. Dudko, A. Ershov, A. Gribushin, V. Klyukhin, O. Kodolova, I. Lokhtin, A. Markina, S. Obraztsov, M. Perfilov, S. Petrushanko, A. Popov,

L. Sarycheva ${ }^{\dagger}$, V. Savrin, A. Snigirev

P.N. Lebedev Physical Institute, Moscow, Russia

V. Andreev, M. Azarkin, I. Dremin, M. Kirakosyan, A. Leonidov, G. Mesyats, S.V. Rusakov, A. Vinogradov 
State Research Center of Russian Federation, Institute for High Energy Physics, Protvino, Russia

I. Azhgirey, I. Bayshev, S. Bitioukov, V. Grishin ${ }^{5}$, V. Kachanov, D. Konstantinov, A. Korablev, V. Krychkine, V. Petrov, R. Ryutin, A. Sobol, L. Tourtchanovitch, S. Troshin, N. Tyurin, A. Uzunian, A. Volkov

University of Belgrade, Faculty of Physics and Vinca Institute of Nuclear Sciences, Belgrade, Serbia

P. Adzic ${ }^{29}$, M. Djordjevic, M. Ekmedzic, D. Krpic ${ }^{29}$, J. Milosevic

Centro de Investigaciones Energéticas Medioambientales y Tecnológicas (CIEMAT), Madrid, Spain

M. Aguilar-Benitez, J. Alcaraz Maestre, P. Arce, C. Battilana, E. Calvo, M. Cerrada, M. Chamizo Llatas, N. Colino, B. De La Cruz, A. Delgado Peris, D. Domínguez Vázquez, C. Fernandez Bedoya, J.P. Fernández Ramos, A. Ferrando, J. Flix, M.C. Fouz, P. GarciaAbia, O. Gonzalez Lopez, S. Goy Lopez, J.M. Hernandez, M.I. Josa, G. Merino, J. Puerta Pelayo, A. Quintario Olmeda, I. Redondo, L. Romero, J. Santaolalla, M.S. Soares, C. Willmott

Universidad Autónoma de Madrid, Madrid, Spain

C. Albajar, G. Codispoti, J.F. de Trocóniz

Universidad de Oviedo, Oviedo, Spain

H. Brun, J. Cuevas, J. Fernandez Menendez, S. Folgueras, I. Gonzalez Caballero, L. Lloret Iglesias, J. Piedra Gomez ${ }^{30}$

Instituto de Física de Cantabria (IFCA), CSIC-Universidad de Cantabria, Santander, Spain

J.A. Brochero Cifuentes, I.J. Cabrillo, A. Calderon, S.H. Chuang, J. Duarte Campderros, M. Felcini ${ }^{31}$, M. Fernandez, G. Gomez, J. Gonzalez Sanchez, C. Jorda, A. Lopez Virto, J. Marco, R. Marco, C. Martinez Rivero, F. Matorras, F.J. Munoz Sanchez, T. Rodrigo, A.Y. Rodríguez-Marrero, A. Ruiz-Jimeno, L. Scodellaro, M. Sobron Sanudo, I. Vila, R. Vilar Cortabitarte

CERN, European Organization for Nuclear Research, Geneva, Switzerland

D. Abbaneo, E. Auffray, G. Auzinger, P. Baillon, A.H. Ball, D. Barney, J.F. Benitez, C. Bernet ${ }^{6}$, G. Bianchi, P. Bloch, A. Bocci, A. Bonato, C. Botta, H. Breuker, T. Camporesi, G. Cerminara, T. Christiansen, J.A. Coarasa Perez, D. D'Enterria, A. Dabrowski, A. De Roeck, S. Di Guida, M. Dobson, N. Dupont-Sagorin, A. Elliott-Peisert, B. Frisch, W. Funk, G. Georgiou, M. Giffels, D. Gigi, K. Gill, D. Giordano, M. Giunta, F. Glege, R. GomezReino Garrido, P. Govoni, S. Gowdy, R. Guida, M. Hansen, P. Harris, C. Hartl, J. Harvey, B. Hegner, A. Hinzmann, V. Innocente, P. Janot, K. Kaadze, E. Karavakis, K. Kousouris, P. Lecoq, Y.-J. Lee, P. Lenzi, C. Lourenço, T. Mäki, M. Malberti, L. Malgeri, M. Mannelli, L. Masetti, F. Meijers, S. Mersi, E. Meschi, R. Moser, M.U. Mozer, M. Mulders, P. Musella, E. Nesvold, T. Orimoto, L. Orsini, E. Palencia Cortezon, E. Perez, L. Perrozzi, A. Petrilli, A. Pfeiffer, M. Pierini, M. Pimiä, D. Piparo, G. Polese, L. Quertenmont, 
A. Racz, W. Reece, J. Rodrigues Antunes, G. Rolandi ${ }^{32}$, T. Rommerskirchen, C. Rovelli ${ }^{33}$, M. Rovere, H. Sakulin, F. Santanastasio, C. Schäfer, C. Schwick, I. Segoni, S. Sekmen, A. Sharma, P. Siegrist, P. Silva, M. Simon, P. Sphicas ${ }^{34}$, D. Spiga, A. Tsirou, G.I. Veres ${ }^{18}$, J.R. Vlimant, H.K. Wöhri, S.D. Worm ${ }^{35}$, W.D. Zeuner

\section{Paul Scherrer Institut, Villigen, Switzerland}

W. Bertl, K. Deiters, W. Erdmann, K. Gabathuler, R. Horisberger, Q. Ingram, H.C. Kaestli, S. König, D. Kotlinski, U. Langenegger, F. Meier, D. Renker, T. Rohe, J. Sibille ${ }^{36}$

Institute for Particle Physics, ETH Zurich, Zurich, Switzerland

L. Bäni, P. Bortignon, M.A. Buchmann, B. Casal, N. Chanon, A. Deisher, G. Dissertori, M. Dittmar, M. Dünser, J. Eugster, K. Freudenreich, C. Grab, D. Hits, P. Lecomte, W. Lustermann, A.C. Marini, P. Martinez Ruiz del Arbol, N. Mohr, F. Moortgat, C. Nägeli ${ }^{37}$, P. Nef, F. Nessi-Tedaldi, F. Pandolfi, L. Pape, F. Pauss, M. Peruzzi, F.J. Ronga, M. Rossini, L. Sala, A.K. Sanchez, A. Starodumov ${ }^{38}$, B. Stieger, M. Takahashi, L. Tauscher ${ }^{\dagger}$, A. Thea, K. Theofilatos, D. Treille, C. Urscheler, R. Wallny, H.A. Weber, L. Wehrli

\section{Universität Zürich, Zurich, Switzerland}

E. Aguilo, C. Amsler, V. Chiochia, S. De Visscher, C. Favaro, M. Ivova Rikova, B. Millan Mejias, P. Otiougova, P. Robmann, H. Snoek, S. Tupputi, M. Verzetti

\section{National Central University, Chung-Li, Taiwan}

Y.H. Chang, K.H. Chen, C.M. Kuo, S.W. Li, W. Lin, Z.K. Liu, Y.J. Lu, D. Mekterovic, A.P. Singh, R. Volpe, S.S. Yu

\section{National Taiwan University (NTU), Taipei, Taiwan}

P. Bartalini, P. Chang, Y.H. Chang, Y.W. Chang, Y. Chao, K.F. Chen, C. Dietz, U. Grundler, W.-S. Hou, Y. Hsiung, K.Y. Kao, Y.J. Lei, R.-S. Lu, D. Majumder, E. Petrakou, X. Shi, J.G. Shiu, Y.M. Tzeng, X. Wan, M. Wang

\section{Cukurova University, Adana, Turkey}

A. Adiguzel, M.N. Bakirci ${ }^{39}$, S. Cerci ${ }^{40}$, C. Dozen, I. Dumanoglu, E. Eskut, S. Girgis, G. Gokbulut, E. Gurpinar, I. Hos, E.E. Kangal, G. Karapinar ${ }^{41}$, A. Kayis Topaksu, G. Onengut, K. Ozdemir, S. Ozturk ${ }^{42}$, A. Polatoz, K. Sogut ${ }^{43}$, D. Sunar Cerci ${ }^{40}$, B. Tali ${ }^{40}$, H. Topakli ${ }^{39}$, L.N. Vergili, M. Vergili

Middle East Technical University, Physics Department, Ankara, Turkey I.V. Akin, T. Aliev, B. Bilin, S. Bilmis, M. Deniz, H. Gamsizkan, A.M. Guler, K. Ocalan, A. Ozpineci, M. Serin, R. Sever, U.E. Surat, M. Yalvac, E. Yildirim, M. Zeyrek

Bogazici University, Istanbul, Turkey

E. Gülmez, B. Isildak ${ }^{44}$, M. Kaya ${ }^{45}$, O. Kaya ${ }^{45}$, S. Ozkorucuklu ${ }^{46}$, N. Sonmez ${ }^{47}$

\section{Istanbul Technical University, Istanbul, Turkey}

K. Cankocak 
National Scientific Center, Kharkov Institute of Physics and Technology, Kharkov, Ukraine

L. Levchuk

\section{University of Bristol, Bristol, United Kingdom}

F. Bostock, J.J. Brooke, E. Clement, D. Cussans, H. Flacher, R. Frazier, J. Goldstein, M. Grimes, G.P. Heath, H.F. Heath, L. Kreczko, S. Metson, D.M. Newbold ${ }^{35}$, K. Nirunpong, A. Poll, S. Senkin, V.J. Smith, T. Williams

\section{Rutherford Appleton Laboratory, Didcot, United Kingdom}

L. Basso ${ }^{48}$, K.W. Bell, A. Belyaev ${ }^{48}$, C. Brew, R.M. Brown, D.J.A. Cockerill, J.A. Coughlan, K. Harder, S. Harper, J. Jackson, B.W. Kennedy, E. Olaiya, D. Petyt, B.C. RadburnSmith, C.H. Shepherd-Themistocleous, I.R. Tomalin, W.J. Womersley

\section{Imperial College, London, United Kingdom}

R. Bainbridge, G. Ball, R. Beuselinck, O. Buchmuller, D. Colling, N. Cripps, M. Cutajar, P. Dauncey, G. Davies, M. Della Negra, W. Ferguson, J. Fulcher, D. Futyan, A. Gilbert, A. Guneratne Bryer, G. Hall, Z. Hatherell, J. Hays, G. Iles, M. Jarvis, G. Karapostoli, L. Lyons, A.-M. Magnan, J. Marrouche, B. Mathias, R. Nandi, J. Nash, A. Nikitenko ${ }^{38}$, A. Papageorgiou, J. Pela ${ }^{5}$, M. Pesaresi, K. Petridis, M. Pioppi ${ }^{49}$, D.M. Raymond, S. Rogerson, A. Rose, M.J. Ryan, C. Seez, P. Sharp ${ }^{\dagger}$, A. Sparrow, M. Stoye, A. Tapper, M. Vazquez Acosta, T. Virdee, S. Wakefield, N. Wardle, T. Whyntie

\section{Brunel University, Uxbridge, United Kingdom}

M. Chadwick, J.E. Cole, P.R. Hobson, A. Khan, P. Kyberd, D. Leggat, D. Leslie, W. Martin, I.D. Reid, P. Symonds, L. Teodorescu, M. Turner

\section{Baylor University, Waco, USA}

K. Hatakeyama, H. Liu, T. Scarborough

The University of Alabama, Tuscaloosa, USA

O. Charaf, C. Henderson, P. Rumerio

\section{Boston University, Boston, USA}

A. Avetisyan, T. Bose, C. Fantasia, A. Heister, J. St. John, P. Lawson, D. Lazic, J. Rohlf, D. Sperka, L. Sulak

\section{Brown University, Providence, USA}

J. Alimena, S. Bhattacharya, D. Cutts, A. Ferapontov, U. Heintz, S. Jabeen, G. Kukartsev, E. Laird, G. Landsberg, M. Luk, M. Narain, D. Nguyen, M. Segala, T. Sinthuprasith, T. Speer, K.V. Tsang

\section{University of California, Davis, Davis, USA}

R. Breedon, G. Breto, M. Calderon De La Barca Sanchez, S. Chauhan, M. Chertok, J. Conway, R. Conway, P.T. Cox, J. Dolen, R. Erbacher, M. Gardner, R. Houtz, W. Ko, A. Kopecky, R. Lander, T. Miceli, D. Pellett, B. Rutherford, M. Searle, J. Smith, M. Squires, M. Tripathi, R. Vasquez Sierra 
University of California, Los Angeles, Los Angeles, USA

V. Andreev, D. Cline, R. Cousins, J. Duris, S. Erhan, P. Everaerts, C. Farrell, J. Hauser, M. Ignatenko, C. Jarvis, C. Plager, G. Rakness, P. Schlein ${ }^{\dagger}$, J. Tucker, V. Valuev, M. Weber University of California, Riverside, Riverside, USA

J. Babb, R. Clare, M.E. Dinardo, J. Ellison, J.W. Gary, F. Giordano, G. Hanson, G.Y. Jeng ${ }^{50}$, H. Liu, O.R. Long, A. Luthra, H. Nguyen, S. Paramesvaran, J. Sturdy, S. Sumowidagdo, R. Wilken, S. Wimpenny

\section{University of California, San Diego, La Jolla, USA}

W. Andrews, J.G. Branson, G.B. Cerati, S. Cittolin, D. Evans, F. Golf, A. Holzner, R. Kelley, M. Lebourgeois, J. Letts, I. Macneill, B. Mangano, S. Padhi, C. Palmer, G. Petrucciani, M. Pieri, M. Sani, V. Sharma, S. Simon, E. Sudano, M. Tadel, Y. Tu, A. Vartak, S. Wasserbaech ${ }^{51}$, F. Würthwein, A. Yagil, J. Yoo

University of California, Santa Barbara, Santa Barbara, USA

D. Barge, R. Bellan, C. Campagnari, M. D’Alfonso, T. Danielson, K. Flowers, P. Geffert, J. Incandela, C. Justus, P. Kalavase, S.A. Koay, D. Kovalskyi, V. Krutelyov, S. Lowette, N. Mccoll, V. Pavlunin, F. Rebassoo, J. Ribnik, J. Richman, R. Rossin, D. Stuart, W. To, C. West

\section{California Institute of Technology, Pasadena, USA}

A. Apresyan, A. Bornheim, Y. Chen, E. Di Marco, J. Duarte, M. Gataullin, Y. Ma, A. Mott, H.B. Newman, C. Rogan, M. Spiropulu ${ }^{4}$, V. Timciuc, P. Traczyk, J. Veverka, R. Wilkinson, Y. Yang, R.Y. Zhu

\section{Carnegie Mellon University, Pittsburgh, USA}

B. Akgun, R. Carroll, T. Ferguson, Y. Iiyama, D.W. Jang, Y.F. Liu, M. Paulini, H. Vogel, I. Vorobiev

\section{University of Colorado at Boulder, Boulder, USA}

J.P. Cumalat, B.R. Drell, C.J. Edelmaier, W.T. Ford, A. Gaz, B. Heyburn, E. Luiggi Lopez, J.G. Smith, K. Stenson, K.A. Ulmer, S.R. Wagner

\section{Cornell University, Ithaca, USA}

J. Alexander, A. Chatterjee, N. Eggert, L.K. Gibbons, B. Heltsley, A. Khukhunaishvili, B. Kreis, N. Mirman, G. Nicolas Kaufman, J.R. Patterson, A. Ryd, E. Salvati, W. Sun, W.D. Teo, J. Thom, J. Thompson, J. Vaughan, Y. Weng, L. Winstrom, P. Wittich

\section{Fairfield University, Fairfield, USA}

D. Winn

\section{Fermi National Accelerator Laboratory, Batavia, USA}

S. Abdullin, M. Albrow, J. Anderson, L.A.T. Bauerdick, A. Beretvas, J. Berryhill, P.C. Bhat, I. Bloch, K. Burkett, J.N. Butler, V. Chetluru, H.W.K. Cheung, F. Chlebana, V.D. Elvira, I. Fisk, J. Freeman, Y. Gao, D. Green, O. Gutsche, J. Hanlon, R.M. Harris, J. Hirschauer, B. Hooberman, S. Jindariani, M. Johnson, U. Joshi, B. Kilminster, B. Klima, S. Kunori, S. Kwan, C. Leonidopoulos, D. Lincoln, R. Lipton, J. Lykken, 
K. Maeshima, J.M. Marraffino, S. Maruyama, D. Mason, P. McBride, K. Mishra, S. Mrenna, Y. Musienko ${ }^{52}$, C. Newman-Holmes, V. O'Dell, O. Prokofyev, E. SextonKennedy, S. Sharma, W.J. Spalding, L. Spiegel, P. Tan, L. Taylor, S. Tkaczyk, N.V. Tran, L. Uplegger, E.W. Vaandering, R. Vidal, J. Whitmore, W. Wu, F. Yang, F. Yumiceva, J.C. Yun

\section{University of Florida, Gainesville, USA}

D. Acosta, P. Avery, D. Bourilkov, M. Chen, S. Das, M. De Gruttola, G.P. Di Giovanni, D. Dobur, A. Drozdetskiy, R.D. Field, M. Fisher, Y. Fu, I.K. Furic, J. Gartner, J. Hugon, B. Kim, J. Konigsberg, A. Korytov, A. Kropivnitskaya, T. Kypreos, J.F. Low, K. Matchev, P. Milenovic ${ }^{53}$, G. Mitselmakher, L. Muniz, R. Remington, A. Rinkevicius, P. Sellers, N. Skhirtladze, M. Snowball, J. Yelton, M. Zakaria

Florida International University, Miami, USA

V. Gaultney, L.M. Lebolo, S. Linn, P. Markowitz, G. Martinez, J.L. Rodriguez

\section{Florida State University, Tallahassee, USA}

J.R. Adams, T. Adams, A. Askew, J. Bochenek, J. Chen, B. Diamond, S.V. Gleyzer, J. Haas, S. Hagopian, V. Hagopian, M. Jenkins, K.F. Johnson, H. Prosper, V. Veeraraghavan, M. Weinberg

\section{Florida Institute of Technology, Melbourne, USA}

M.M. Baarmand, B. Dorney, M. Hohlmann, H. Kalakhety, I. Vodopiyanov

\section{University of Illinois at Chicago (UIC), Chicago, USA}

M.R. Adams, I.M. Anghel, L. Apanasevich, Y. Bai, V.E. Bazterra, R.R. Betts, I. Bucinskaite, J. Callner, R. Cavanaugh, C. Dragoiu, O. Evdokimov, L. Gauthier, C.E. Gerber, D.J. Hofman, S. Khalatyan, F. Lacroix, M. Malek, C. O'Brien, C. Silkworth, D. Strom, N. Varelas

\section{The University of Iowa, Iowa City, USA}

U. Akgun, E.A. Albayrak, B. Bilki ${ }^{54}$, W. Clarida, F. Duru, S. Griffiths, J.-P. Merlo, H. Mermerkaya ${ }^{55}$, A. Mestvirishvili, A. Moeller, J. Nachtman, C.R. Newsom, E. Norbeck, Y. Onel, F. Ozok, S. Sen, E. Tiras, J. Wetzel, T. Yetkin, K. Yi

\section{Johns Hopkins University, Baltimore, USA}

B.A. Barnett, B. Blumenfeld, S. Bolognesi, D. Fehling, G. Giurgiu, A.V. Gritsan, Z.J. Guo, G. Hu, P. Maksimovic, S. Rappoccio, M. Swartz, A. Whitbeck

\section{The University of Kansas, Lawrence, USA}

P. Baringer, A. Bean, G. Benelli, O. Grachov, R.P. Kenny Iii, M. Murray, D. Noonan, S. Sanders, R. Stringer, G. Tinti, J.S. Wood, V. Zhukova

\section{Kansas State University, Manhattan, USA}

A.F. Barfuss, T. Bolton, I. Chakaberia, A. Ivanov, S. Khalil, M. Makouski, Y. Maravin, S. Shrestha, I. Svintradze

\section{Lawrence Livermore National Laboratory, Livermore, USA}

J. Gronberg, D. Lange, D. Wright 


\section{University of Maryland, College Park, USA}

A. Baden, M. Boutemeur, B. Calvert, S.C. Eno, J.A. Gomez, N.J. Hadley, R.G. Kellogg, M. Kirn, T. Kolberg, Y. Lu, M. Marionneau, A.C. Mignerey, K. Pedro, A. Peterman, A. Skuja, J. Temple, M.B. Tonjes, S.C. Tonwar, E. Twedt

\section{Massachusetts Institute of Technology, Cambridge, USA}

A. Apyan, G. Bauer, J. Bendavid, W. Busza, E. Butz, I.A. Cali, M. Chan, V. Dutta, G. Gomez Ceballos, M. Goncharov, K.A. Hahn, Y. Kim, M. Klute, K. Krajczar ${ }^{56}$, W. Li, P.D. Luckey, T. Ma, S. Nahn, C. Paus, D. Ralph, C. Roland, G. Roland, M. Rudolph, G.S.F. Stephans, F. Stöckli, K. Sumorok, K. Sung, D. Velicanu, E.A. Wenger, R. Wolf, B. Wyslouch, S. Xie, M. Yang, Y. Yilmaz, A.S. Yoon, M. Zanetti

\section{University of Minnesota, Minneapolis, USA}

S.I. Cooper, B. Dahmes, A. De Benedetti, G. Franzoni, A. Gude, S.C. Kao, K. Klapoetke, Y. Kubota, J. Mans, N. Pastika, R. Rusack, M. Sasseville, A. Singovsky, N. Tambe, J. Turkewitz

\section{University of Mississippi, University, USA}

L.M. Cremaldi, R. Kroeger, L. Perera, R. Rahmat, D.A. Sanders

University of Nebraska-Lincoln, Lincoln, USA

E. Avdeeva, K. Bloom, S. Bose, J. Butt, D.R. Claes, A. Dominguez, M. Eads, J. Keller, I. Kravchenko, J. Lazo-Flores, H. Malbouisson, S. Malik, G.R. Snow

\section{State University of New York at Buffalo, Buffalo, USA}

U. Baur, A. Godshalk, I. Iashvili, S. Jain, A. Kharchilava, A. Kumar, S.P. Shipkowski, K. Smith

\section{Northeastern University, Boston, USA}

G. Alverson, E. Barberis, D. Baumgartel, M. Chasco, J. Haley, D. Nash, D. Trocino, D. Wood, J. Zhang

\section{Northwestern University, Evanston, USA}

A. Anastassov, A. Kubik, N. Mucia, N. Odell, R.A. Ofierzynski, B. Pollack, A. Pozdnyakov, M. Schmitt, S. Stoynev, M. Velasco, S. Won

\section{University of Notre Dame, Notre Dame, USA}

L. Antonelli, D. Berry, A. Brinkerhoff, M. Hildreth, C. Jessop, D.J. Karmgard, J. Kolb, K. Lannon, W. Luo, S. Lynch, N. Marinelli, D.M. Morse, T. Pearson, R. Ruchti, J. Slaunwhite, N. Valls, M. Wayne, M. Wolf

\section{The Ohio State University, Columbus, USA}

B. Bylsma, L.S. Durkin, A. Hart, C. Hill, R. Hughes, K. Kotov, T.Y. Ling, D. Puigh, M. Rodenburg, C. Vuosalo, G. Williams, B.L. Winer

\section{Princeton University, Princeton, USA}

N. Adam, E. Berry, P. Elmer, D. Gerbaudo, V. Halyo, P. Hebda, J. Hegeman, A. Hunt, P. Jindal, D. Lopes Pegna, P. Lujan, D. Marlow, T. Medvedeva, M. Mooney, J. Olsen, 
P. Piroué, X. Quan, A. Raval, B. Safdi, H. Saka, D. Stickland, C. Tully, J.S. Werner, A. Zuranski

\section{University of Puerto Rico, Mayaguez, USA}

J.G. Acosta, E. Brownson, X.T. Huang, A. Lopez, H. Mendez, S. Oliveros, J.E. Ramirez Vargas, A. Zatserklyaniy

Purdue University, West Lafayette, USA

E. Alagoz, V.E. Barnes, D. Benedetti, G. Bolla, D. Bortoletto, M. De Mattia, A. Everett, Z. Hu, M. Jones, O. Koybasi, M. Kress, A.T. Laasanen, N. Leonardo, V. Maroussov, P. Merkel, D.H. Miller, N. Neumeister, I. Shipsey, D. Silvers, A. Svyatkovskiy, M. Vidal Marono, H.D. Yoo, J. Zablocki, Y. Zheng

Purdue University Calumet, Hammond, USA

S. Guragain, N. Parashar

\section{Rice University, Houston, USA}

A. Adair, C. Boulahouache, K.M. Ecklund, F.J.M. Geurts, B.P. Padley, R. Redjimi, J. Roberts, J. Zabel

\section{University of Rochester, Rochester, USA}

B. Betchart, A. Bodek, Y.S. Chung, R. Covarelli, P. de Barbaro, R. Demina, Y. Eshaq, A. Garcia-Bellido, P. Goldenzweig, J. Han, A. Harel, D.C. Miner, D. Vishnevskiy, M. Zielinski

\section{The Rockefeller University, New York, USA}

A. Bhatti, R. Ciesielski, L. Demortier, K. Goulianos, G. Lungu, S. Malik, C. Mesropian

Rutgers, the State University of New Jersey, Piscataway, USA

S. Arora, A. Barker, J.P. Chou, C. Contreras-Campana, E. Contreras-Campana, D. Duggan, D. Ferencek, Y. Gershtein, R. Gray, E. Halkiadakis, D. Hidas, A. Lath, S. Panwalkar, M. Park, R. Patel, V. Rekovic, J. Robles, K. Rose, S. Salur, S. Schnetzer, C. Seitz, S. Somalwar, R. Stone, S. Thomas

\section{University of Tennessee, Knoxville, USA}

G. Cerizza, M. Hollingsworth, S. Spanier, Z.C. Yang, A. York

\section{Texas A\&M University, College Station, USA}

R. Eusebi, W. Flanagan, J. Gilmore, T. Kamon ${ }^{57}$, V. Khotilovich, R. Montalvo, I. Osipenkov, Y. Pakhotin, A. Perloff, J. Roe, A. Safonov, T. Sakuma, S. Sengupta, I. Suarez, A. Tatarinov, D. Toback

\section{Texas Tech University, Lubbock, USA}

N. Akchurin, J. Damgov, P.R. Dudero, C. Jeong, K. Kovitanggoon, S.W. Lee, T. Libeiro, Y. Roh, I. Volobouev

\section{Vanderbilt University, Nashville, USA}

E. Appelt, C. Florez, S. Greene, A. Gurrola, W. Johns, C. Johnston, P. Kurt, C. Maguire, A. Melo, P. Sheldon, B. Snook, S. Tuo, J. Velkovska 


\section{University of Virginia, Charlottesville, USA}

M.W. Arenton, M. Balazs, S. Boutle, B. Cox, B. Francis, J. Goodell, R. Hirosky, A. Ledovskoy, C. Lin, C. Neu, J. Wood, R. Yohay

\section{Wayne State University, Detroit, USA}

S. Gollapinni, R. Harr, P.E. Karchin, C. Kottachchi Kankanamge Don, P. Lamichhane, A. Sakharov

\section{University of Wisconsin, Madison, USA}

M. Anderson, M. Bachtis, D. Belknap, L. Borrello, D. Carlsmith, M. Cepeda, S. Dasu, L. Gray, K.S. Grogg, M. Grothe, R. Hall-Wilton, M. Herndon, A. Hervé, P. Klabbers, J. Klukas, A. Lanaro, C. Lazaridis, J. Leonard, R. Loveless, A. Mohapatra, I. Ojalvo, F. Palmonari, G.A. Pierro, I. Ross, A. Savin, W.H. Smith, J. Swanson

$\dagger$ : Deceased

1: Also at Vienna University of Technology, Vienna, Austria

2: Also at National Institute of Chemical Physics and Biophysics, Tallinn, Estonia

3: Also at Universidade Federal do ABC, Santo Andre, Brazil

4: Also at California Institute of Technology, Pasadena, USA

5: Also at CERN, European Organization for Nuclear Research, Geneva, Switzerland

6: Also at Laboratoire Leprince-Ringuet, Ecole Polytechnique, IN2P3-CNRS, Palaiseau, France

7: Also at Suez Canal University, Suez, Egypt

8: Also at Zewail City of Science and Technology, Zewail, Egypt

9: Also at Cairo University, Cairo, Egypt

10: Also at Fayoum University, El-Fayoum, Egypt

11: Also at British University, Cairo, Egypt

12: Now at Ain Shams University, Cairo, Egypt

13: Also at Soltan Institute for Nuclear Studies, Warsaw, Poland

14: Also at Université de Haute-Alsace, Mulhouse, France

15: Also at Moscow State University, Moscow, Russia

16: Also at Brandenburg University of Technology, Cottbus, Germany

17: Also at Institute of Nuclear Research ATOMKI, Debrecen, Hungary

18: Also at Eötvös Loránd University, Budapest, Hungary

19: Also at Tata Institute of Fundamental Research - HECR, Mumbai, India

20: Also at University of Visva-Bharati, Santiniketan, India

21: Also at Sharif University of Technology, Tehran, Iran

22: Also at Isfahan University of Technology, Isfahan, Iran

23: Also at Plasma Physics Research Center, Science and Research Branch, Islamic Azad University, Teheran, Iran

24: Also at Facoltà Ingegneria Università di Roma, Roma, Italy

25: Also at Università della Basilicata, Potenza, Italy

26: Also at Università degli Studi Guglielmo Marconi, Roma, Italy

27: Also at Università degli studi di Siena, Siena, Italy

28: Also at University of Bucharest, Faculty of Physics, Bucuresti-Magurele, Romania

29: Also at Faculty of Physics of University of Belgrade, Belgrade, Serbia

30: Also at University of Florida, Gainesville, USA

31: Also at University of California, Los Angeles, Los Angeles, USA 
32: Also at Scuola Normale e Sezione dell' INFN, Pisa, Italy

33: Also at INFN Sezione di Roma; Università di Roma "La Sapienza", Roma, Italy

34: Also at University of Athens, Athens, Greece

35: Also at Rutherford Appleton Laboratory, Didcot, United Kingdom

36: Also at The University of Kansas, Lawrence, USA

37: Also at Paul Scherrer Institut, Villigen, Switzerland

38: Also at Institute for Theoretical and Experimental Physics, Moscow, Russia

39: Also at Gaziosmanpasa University, Tokat, Turkey

40: Also at Adiyaman University, Adiyaman, Turkey

41: Also at Izmir Institute of Technology, Izmir, Turkey

42: Also at The University of Iowa, Iowa City, USA

43: Also at Mersin University, Mersin, Turkey

44: Also at Ozyegin University, Istanbul, Turkey

45: Also at Kafkas University, Kars, Turkey

46: Also at Suleyman Demirel University, Isparta, Turkey

47: Also at Ege University, Izmir, Turkey

48: Also at School of Physics and Astronomy, University of Southampton, Southampton, United Kingdom

49: Also at INFN Sezione di Perugia; Università di Perugia, Perugia, Italy

50: Also at University of Sydney, Sydney, Australia

51: Also at Utah Valley University, Orem, USA

52: Also at Institute for Nuclear Research, Moscow, Russia

53: Also at University of Belgrade, Faculty of Physics and Vinca Institute of Nuclear Sciences, Belgrade, Serbia

54: Also at Argonne National Laboratory, Argonne, USA

55: Also at Erzincan University, Erzincan, Turkey

56: Also at KFKI Research Institute for Particle and Nuclear Physics, Budapest, Hungary

57: Also at Kyungpook National University, Daegu, Korea 